S. Akiyama and Y. Tanigawa

Nagoya Math. J.

Vol. 117 (1990), 93-123

\title{
THE SELBERG TRACE FORMULA FOR MODULAR CORRESPONDENCES
}

\author{
SHIGEKI AKIYAMA AND YOSHIO TANIGAWA
}

\section{§ 0. Introduction}

In Selberg [11], he introduced the trace formula and applied it to computations of traces of Hecke operators acting on the space of cusp forms of weight greater than or equal to two. But for the case of weight one, the similar method is not effective. It only gives us a certain expression of the dimension of the space of cusp forms by the residue of the Selberg type zeta function. Here the Selberg type zeta function appears in the contribution from the hyperbolic conjugacy classes when we write the trace formula with a certain kernel function ([3], [4], [7], [8], [9], [12]).

The starting point of the present work is [2], where we treated the trace formula of weight one for modular correspondences with the same kernel function and expressed the trace of the Hecke operator by the residue of the corresponding Selberg type zeta function. Now we note that the above Selberg type zeta function does not have a functional equation. This is because the poles of the Selberg transform $h_{s}(r)$ are not placed symmetrically.

In this paper, we take up our problem again and consider the trace formula for modular correspondences with a general kernel function. It was already done in Hejhal [5] Chapter 5, when $\Gamma$ has a compact fundamental domain. We only assume here that $\Gamma$ is a Fuchsian group of the first kind. There may exist, in a double coset of $\Gamma$, hyperbolic conjugacy classes whose fixed points are cusps and parabolic conjugacy classes. The contributions from these terms are new points of this paper. In $\S 1$, we introduce the necessary notation for the Selberg trace formula. In $\S 2-\S 6$, we calculate the contribution from each conjugacy class. In the last section, we restrict ourselves to the case $\Gamma=\Gamma_{0}(p)$ and calculate the terms in $\S 4-\S 6$ as explicitly as possible. We also define the Selberg type

Received December 5, 1988. 
zeta function for a double coset by taking the classical Selberg kernel as the kernel function and prove the functional equation (cf. [1]).

\section{§ 1. Preliminary}

Let $\Gamma$ be a Fuchsian group of the first kind of $G=S L_{2}(R)$ which does not contain -1 , and $\bar{\Gamma}$ be the commensurator of $\Gamma$ in $G$. Take an element $\alpha$ from $\bar{\Gamma} \backslash\{-1\} \cdot \Gamma$. Let $\boldsymbol{H}$ be the complex upper half plane and $\chi$ be a unitary representation of degree $\nu$ of the group generated by $\Gamma$ and $\alpha$. If $\Gamma$ is not cocompact then we assume that the values of $\chi$ at the stabilizer of each cusp are the roots of the unit matrix. Let $f$ be a holomorphic mapping from $\boldsymbol{H}$ to $\boldsymbol{C}^{\nu}$ satisfying

$$
f \mid[\gamma]_{m}=\chi(\gamma) f
$$

$$
f \text { vanishes at each cusp of } \Gamma
$$

where $\left(f \mid[\gamma]_{m}\right)(z)=j(\gamma, z)^{-m} f(\gamma \cdot z), j(\gamma, z)=(c z+d)$ and $\gamma=\left(\begin{array}{ll}a & b \\ c & d\end{array}\right) \in \Gamma$. Denote by $S_{m}(\Gamma, \chi)$ the space of these mappings. Hecke operator which acts on $S_{m}(\Gamma, \chi)$ is defined by

$$
T(\Gamma \alpha \Gamma) \cdot f(z)=\sum \chi\left(\alpha_{\mu}\right) f \mid\left[\alpha_{\mu}^{-1}\right]_{m}
$$

where $\Gamma \alpha \Gamma=\cup_{\mu} \alpha_{\mu} \Gamma$ (disjoint). The trace of $T(\Gamma \alpha \Gamma)$ is calculated by the Selberg trace formula in various cases and used in the arithmetical problems.

Let us review our situation. Put $\tilde{G}=G \times T, \tilde{\Gamma}=\Gamma \times T$ and $\tilde{\boldsymbol{H}}=\boldsymbol{H}$ $\times T$, where $T=R / 2 \pi Z$. Then $\tilde{G}$ acts transitively on $\tilde{H}$ by

$$
(g, \theta) \cdot(z, \phi)=(g \cdot z, \phi+\operatorname{Arg}(j(g, z))-\theta) .
$$

The algebra of $\tilde{G}$-invariant differential operators is generated by

$$
\Delta=y^{2}\left(\frac{\partial^{2}}{\partial x^{2}}+\frac{\partial^{2}}{\partial y^{2}}\right)+y \frac{\partial}{\partial x} \frac{\partial}{\partial \phi}, \text { and } \frac{\partial}{\partial \phi} .
$$

Identify $\Gamma$ with $\Gamma \times\{0\}, G$ with $G \times\{0\}$. Let $L_{\chi}^{2}(\tilde{\Gamma} \backslash \tilde{\boldsymbol{H}})$ be the space of mappings from $\tilde{\boldsymbol{H}}$ to $\boldsymbol{C}^{\nu}$ satisfying three conditions:

$$
\begin{aligned}
& F(z, \phi)={ }^{t}\left(f_{1}, f_{2}, \cdots, f_{\nu}\right) \text { is a column vector and each } f_{i} \text { is a } \\
& \text { measurable function on } \tilde{\boldsymbol{H}} \text {, }
\end{aligned}
$$

$$
F(\gamma \cdot(z, \phi))=\chi(\gamma) F(z, \phi) \quad \text { for all } \gamma \in \Gamma,
$$




$$
\int_{\Gamma \backslash \hat{H}}{ }^{t} F(z, \phi) \overline{F(z, \phi)} d z d \phi<\infty .
$$

The Selberg eigenspace $L_{x}(m, \lambda)$ is the subspace of $L_{x}^{2}(\tilde{\Gamma} \backslash \boldsymbol{H})$ with two additional conditions:

$$
\begin{gathered}
\frac{\partial}{\partial \phi} F=-\sqrt{-1} m F, \\
\Delta F=\lambda F .
\end{gathered}
$$

The eigenvalues $\lambda$ are numbered as

$$
\frac{|m|}{2}\left(\frac{|m|}{2}-1\right) \geq \lambda_{1} \geq \lambda_{2} \geq \cdots .
$$

For the convenience, we put $\lambda=s(s-1)=-1 / 4-r^{2}$. Let $\mathscr{S}_{m}(\Gamma, \chi)$ be the space of holomorphic mappings from $\boldsymbol{H}$ to $\boldsymbol{C}^{\nu}$ which satisfy (0) and

$$
\int_{\Gamma \backslash \boldsymbol{H}}{ }^{t} f(z) \overline{f(z)} y^{m} d z<\infty .
$$

If $m \geq 1$ then $\mathscr{S}_{m}(\Gamma, \chi)=S_{m}(\Gamma, \chi)$, and if $m<0$ then $\mathscr{S}_{m}(\Gamma, \chi)=\{0\}$. Note the fact that $\mathscr{S}_{0}(\Gamma$, id) is the space of constant functions. The relation between $\mathscr{S}_{m}(\Gamma, \chi)$ and $L_{\mathrm{x}}(m, \lambda)$ is given by the next lemma.

LEMma 1. We have

$$
\begin{aligned}
& L_{\chi}\left(m+2, \frac{m}{2}\left(1+\frac{m}{2}\right)\right)=y^{(m+2) / 2} \exp (-\sqrt{-1}(m+2) \phi) \mathscr{S}_{m+2}(\Gamma, \chi) \\
& L_{\chi}\left(m, \frac{m}{2}\left(1+\frac{m}{2}\right)\right)=y^{-m / 2} \exp (-\sqrt{-1} m \phi) \overline{\mathscr{S}_{-m}(\Gamma, \bar{\chi})} .
\end{aligned}
$$

For the proof of this lemma, see Hejhal [6] p. 383. The Hecke operator on $L_{x}^{2}(\tilde{\Gamma} \backslash \tilde{\boldsymbol{H}})$ is defined by

$$
T(\Gamma \alpha \Gamma) F(z, \phi)=\sum_{\mu} \chi\left(\alpha_{\mu}\right) F\left(\alpha_{\mu}^{-1} \cdot(z, \phi)\right) .
$$

This definition is compatible with the former one. Denote by $\operatorname{tr}\left(T(\Gamma \alpha \Gamma) ; \lambda_{j}\right)$ the trace of $T(\Gamma \alpha \Gamma)$ acting on $L_{x}\left(m, \lambda_{j}\right)$. Put

(8) $\quad k\left(z, \phi, z^{\prime}, \phi^{\prime}\right)=\Phi\left(\frac{\left|z-z^{\prime}\right|^{2}}{y y^{\prime}}\right) \exp \left(-\sqrt{-1} m\left(\operatorname{Arg}\left(\frac{z-\bar{z}^{\prime}}{2 \sqrt{-1}}\right)+\phi-\phi^{\prime}\right)\right)$

where $y=\operatorname{Im}(z), y^{\prime}=\operatorname{Im}\left(z^{\prime}\right)$, and $\Phi$ is the $C^{4}$-class function from $(0, \infty)$ to $(0, \infty)$ such that 


$$
\left|\Phi^{(k)}(t)\right|<A(t+1)^{-\tau-k}
$$

where $\tau>\max \{|m / 2|, 1\}, \quad k=0,1,2,3,4$, and $A$ is a positive constant. This integral kernel is a point pair invariant, i.e.

$$
k\left(g \cdot(z, \phi), g \cdot\left(z^{\prime}, \phi^{\prime}\right)\right)=k\left(z, \phi, z^{\prime}, \phi^{\prime}\right)
$$

for all $g \in G$. The eigenvalue of the integral operator

$$
F(z, \phi) \longrightarrow \frac{1}{2 \pi} \int_{\ddot{\theta}} k\left(z, \phi, z^{\prime}, \phi^{\prime}\right) F\left(z^{\prime}, \phi^{\prime}\right) d z^{\prime} d \phi^{\prime}
$$

is independent of the choice of elements in $L_{x}(m, \lambda)$. (For the consistency with the notation of the work of Hejhal [6], the constant factor $1 / 2 \pi$ is multiplied.) The above integral vanishes when $F \in L_{x}\left(m^{\prime}, \lambda\right)$ and $m \neq m^{\prime}$. We denote this eigenvalue by $\Lambda(s(s-1))=h(r)$. Then the correspondence between $\Phi$ and $\Lambda$ is given by the following equations (9) (14) (See [6], p. 386).

$$
\begin{aligned}
& \Lambda(s(s-1)) \exp (-\sqrt{-1} m \phi) \\
& =\frac{1}{2 \pi} \int_{\tilde{\boldsymbol{H}}} k\left(\sqrt{-1}, \phi, z^{\prime}, \phi^{\prime}\right) y^{\prime s} \exp \left(-\sqrt{-1} m \phi^{\prime}\right) d z^{\prime} d \phi^{\prime} . \\
& Q(w)=\int_{-\infty}^{\infty} \Phi\left(w+v^{2}\right)\left(\frac{\sqrt{w+4}+\sqrt{-1} v}{\sqrt{w+4}-\sqrt{-1} v}\right)^{m / 2} d v \\
& \Phi(x)=\int_{-\infty}^{\infty} Q^{\prime}\left(x+t^{2}\right)\left(\frac{\sqrt{x+4+t^{2}}-\sqrt{-1} t}{\sqrt{x+4+t^{2}}+\sqrt{-1} t}\right)^{m / 2} d t
\end{aligned}
$$

where $Q^{\prime}$ is the derivative of $Q$ and

$$
\begin{gathered}
g(u)=Q\left(e^{u}+e^{-u}-2\right) \\
\Lambda(s(s-1))=h(r)=\int_{-\infty}^{\infty} g(u) \exp (\sqrt{-1} r u) d u .
\end{gathered}
$$

So $g(u)$ is represented by $h(r)$ :

$$
g(u)=\frac{1}{2 \pi} \int_{-\infty}^{\infty} h(r) \exp (-\sqrt{-1} r u) d r .
$$

Now we describe the Selberg trace formula for modular correspondences in the cocompact case. Let $F(z, \phi)$ be an element of $L_{x}(m, \lambda)$, then

$$
\Lambda(s(s-1)) F(z, \phi)=\frac{1}{2 \pi} \int_{\tilde{H}} k\left(z, \phi, z^{\prime}, \phi^{\prime}\right) F\left(z^{\prime}, \phi^{\prime}\right) d z^{\prime} d \phi^{\prime} .
$$


Thus

$$
\begin{aligned}
h(r) T(\Gamma \alpha \Gamma) F(z, \phi) & =\frac{1}{2 \pi} \int_{\tilde{H}} \sum_{\mu} \chi\left(\alpha_{\mu}\right) k\left(\alpha_{\mu}^{-1}(z, \phi), z^{\prime}, \phi^{\prime}\right) F\left(z^{\prime}, \phi^{\prime}\right) d z^{\prime} d \phi^{\prime} \\
& =\frac{1}{2 \pi} \int_{\Gamma \backslash \tilde{H}} K\left(z, \phi, z^{\prime}, \phi^{\prime}\right) F\left(z^{\prime}, \phi^{\prime}\right) d z^{\prime} d \phi^{\prime}
\end{aligned}
$$

where $K\left(z, \phi, z^{\prime}, \phi\right)=\sum_{g \in \Gamma \alpha \Gamma} \chi(g) k\left(z, \phi, g\left(z^{\prime}, \phi^{\prime}\right)\right)$.

Taking traces of both sides, we see that

$$
\begin{gathered}
\sum_{j} h\left(\sqrt{-1 / 4-\lambda_{j}}\right) \operatorname{tr}\left(T(\Gamma \alpha \Gamma) ; \lambda_{\jmath}\right)=\frac{1}{2 \pi} \int_{\Gamma \backslash \tilde{\boldsymbol{H}}} \operatorname{tr} K(z, \phi, z, \phi) d z d \phi \\
=\frac{1}{2 \pi} \sum_{[g]} \operatorname{tr} \chi(g) \int_{\Gamma^{(}(g) \backslash \tilde{\boldsymbol{B}}} k(z, \phi, g(z, \phi)) d z d \phi
\end{gathered}
$$

where [ ] is the conjugacy class of $\Gamma \alpha \Gamma$ with respect to $\Gamma$, and $\Gamma(g)$ is the centralizer of $g$ in $\Gamma$. In the case that $\Gamma$ is not cocompact, the integral of the right hand side diverges by the effect of continuous spectra. Hence we must subtract this effect. For this purpose, we define the Eisenstein series for each cusp of $\tilde{\Gamma} \backslash \tilde{\boldsymbol{H}}$. Let $\kappa_{1}, \kappa_{2}, \cdots, \kappa_{\omega}$ be the complete representatives of $\Gamma$-inequivalent cusps of $\Gamma \backslash \boldsymbol{H}$. We denote $\Gamma_{i}$ the stabilizer of $\kappa_{i}$, and $\Gamma_{i}^{0}=\Gamma_{i} \cap \operatorname{ker} \chi$. Take $\sigma_{i} \in G$ such that $\sigma_{i} \infty=\kappa_{i}$, satisfying the following condition.

$$
\left\{\begin{array}{l}
\text { If } \kappa_{i} \text { is regular then } \Gamma_{\infty}=\sigma_{i}^{-1} \Gamma_{i} \sigma_{i} \text { is generated by }\left(\begin{array}{ll}
1 & 1 \\
0 & 1
\end{array}\right), \\
\text { If } \kappa_{i} \text { is irregular then } \Gamma_{\infty}=\sigma_{i}^{-1} \Gamma_{i} \sigma_{i} \text { is generated by }\left(\begin{array}{rr}
-1 & -1 \\
0 & -1
\end{array}\right) .
\end{array}\right.
$$

Define the Eisenstein series for $\kappa_{i}(i=1, \cdots, \omega)$ by

$$
\begin{aligned}
E_{i}(z, \phi ; s) & =\sum_{\sigma \in \Gamma_{i} \backslash \Gamma} \operatorname{Im}\left(\sigma_{i}^{-1} \sigma z\right)^{s} \exp \left(-m \sqrt{-1}\left(\phi+\operatorname{Arg} j\left(\sigma_{i}^{-1} \sigma, z\right)\right)\right) \chi^{-1}(\sigma) P_{i} \\
& =\sum_{\sigma \in \Gamma_{\infty} \backslash \sigma_{i}^{-1} \Gamma} \frac{y^{s}}{|c z+d|^{2 s}} \exp (-m \sqrt{-1}(\phi+\operatorname{Arg}(c z+d))) \chi^{-1}\left(\sigma_{i} \sigma\right) P_{i} .
\end{aligned}
$$

where $\sigma=\left(\begin{array}{ll}a & b \\ c & d\end{array}\right) . \quad P_{i}$ is defined by

$$
P_{i}= \begin{cases}\frac{1}{2 r_{i}} \sum_{k=1}^{2 r_{i}}(-1)^{k} \chi\left(\eta^{k}\right) & \text { if } m \text { is odd and } \kappa_{i} \text { is irregular } \\ \frac{1}{r_{i}} \sum_{g \in \Gamma_{i} / \Gamma_{i}^{0}} \chi(g) & \text { otherwise }\end{cases}
$$


where $r_{i}=\left[\Gamma_{i}: \Gamma_{i}^{0}\right]$ and $\eta \in \Gamma_{i}$ is chosen so that $\eta \bmod \left(\Gamma_{i}^{0}\right)^{2}$ should be a generator of $\Gamma_{i} /\left(\Gamma_{i}^{0}\right)^{2}$ (c.f. Ishikawa [9]). Each Eisenstein series is meromorphically continued to the whole $s$-plane and satisfies a certain functional equation. Put

$$
H\left(z, \phi, z^{\prime}, \phi^{\prime}\right)=\frac{1}{4 \pi} \sum_{i=1}^{\omega} \sum_{\mu} \chi\left(\alpha_{\mu}\right) \int_{-\infty}^{\infty} h(r) E_{i}\left(\alpha_{\mu}^{-1}(z, \phi), s\right)^{t} \overline{E_{i}\left(z^{\prime}, \phi^{\prime}, s\right)} d r,
$$

where the sum over $\alpha_{\mu}$ is taken over all representatives of conjugacy classes of $\Gamma \alpha \Gamma$ which fix cusps of $\Gamma$, and $s=1 / 2+\sqrt{-1} r$.

Lemma 2. The function $K\left(z, \phi, z^{\prime}, \phi^{\prime}\right)-H\left(z, \phi, z^{\prime}, \phi^{\prime}\right)$ is bounded in $\left(z, \phi, z^{\prime}, \phi^{\prime}\right) \in \Gamma \backslash \tilde{\boldsymbol{H}} \times \Gamma \backslash \tilde{\boldsymbol{H}}$.

Proof. We must consider when both $z$ and $z^{\prime}$ approach to a $\Gamma$-equivalent cusp of $\tilde{\Gamma} \backslash \tilde{\boldsymbol{H}}$. For simplicity, we assume that $\kappa_{1}=\infty$ is the regular cusp of $\Gamma$, and $z, z^{\prime}$ are in the neighborhood of $\kappa_{1}$. Using Maass-Selberg relation, we have

$$
\begin{aligned}
H(z, \phi, & \left.z^{\prime}, \phi^{\prime}\right)=\sum_{\mu} \frac{\chi\left(\alpha_{\mu}\right)}{4 \pi} \int_{-\infty}^{\infty} h(r)\left(\sum_{i=1}^{\omega} E_{i}\left(\alpha_{\mu}^{-1}(z, \phi) ; s\right)^{t} \overline{E_{i}\left(z^{\prime}, \phi^{\prime}, s\right)}\right) d r \\
= & \exp \left(-m \sqrt{-1}\left(\phi_{1}-\phi^{\prime}\right)\right) \sum_{\mu} \frac{\chi\left(\alpha_{\mu}\right)}{4 \pi} \int_{-\infty}^{\infty} h(r)\left(\sum_{i=1}^{\omega}\left\{y_{1}^{s} \delta_{i 1} P_{1}+y_{1}^{1-s} \varphi_{i 1}(s)\right\}\right. \\
& \left.\times\left\{y_{2}^{\bar{s}} \delta_{i 1}^{t} \bar{P}_{1}+y_{2}^{1-\bar{s}} \overline{\varphi_{i 1}(s)}\right\}\right) d r+O(1) \\
= & \exp \left(-m \sqrt{-1}\left(\phi_{1}-\phi^{\prime}\right)\right) \sum_{\mu} \frac{\chi\left(\alpha_{\mu}\right) \sqrt{y_{1} y_{2}}}{4 \pi} \\
& \times P_{1} \int_{-\infty}^{\infty} h(r)\left(\left(y_{1} / y_{2}\right)^{\sqrt{-1} r}+\left(y_{2} / y_{1}\right)^{\sqrt{-1} r}\right) d r+O(1) \\
= & \exp \left(-m \sqrt{-1}\left(\phi_{1}-\phi^{\prime}\right)\right) \sum_{\mu} \chi\left(\alpha_{\mu}\right) \sqrt{y_{1} y_{2}} g\left(\log y_{1}-\log y_{2}\right) P_{1}+O(1),
\end{aligned}
$$

where $\alpha_{\mu}^{-1} z=x_{1}+\sqrt{-1} y_{1}, \phi_{1}=\phi+\arg j\left(\alpha_{\mu}^{-1}, z\right), z^{\prime}=x_{2}+\sqrt{-1} y_{2}, O(1)$ is the Landau large $O$-symbol with respect to $y_{1}, y_{2} \rightarrow \infty$, and $\varphi_{i j}(s)$ appears in the constant term of the Fourier-Bessel expansion of the Eisenstein series which will be defined and calculated in $\S 6$, Lemma 6. About $K\left(z, \phi, z^{\prime}, \phi^{\prime}\right)$, we get

$$
\begin{aligned}
K\left(z, \phi, z^{\prime}, \phi^{\prime}\right) \\
\quad=\sum_{g \in \Gamma \alpha \Gamma} \chi(g) k\left(z, \phi, g\left(z^{\prime}, \phi^{\prime}\right)\right) \\
\quad=\sum_{\mu} \chi\left(\alpha_{\mu}\right) \sum_{k=0}^{r_{i}-1} \chi\left(\left(\begin{array}{ll}
1 & k \\
0 & 1
\end{array}\right)\right) \sum_{t \in Z} k\left(\alpha_{\mu}^{-1}(z, \phi), z^{\prime}+k+r_{i} t, \phi^{\prime}\right)+O(1) .
\end{aligned}
$$


Using Euler-Maclaurin summation formula, we have

$$
\begin{aligned}
& \sum_{t \in Z} k\left(\alpha_{\mu}^{-1}(z, \phi), z^{\prime}+k+r_{i} t, \phi^{\prime}\right) \\
& =\frac{1}{r_{i}} \int_{-\infty}^{\infty} k\left(\left(\sqrt{-1} y_{1}, \phi_{1}\right), t+\sqrt{-1} y_{2}, \phi^{\prime}\right) d t+O(1) \\
& =\frac{1}{r_{i}} \int_{-\infty}^{\infty} \Phi\left(\frac{\left(y_{1}-y_{2}\right)^{2} t^{2}}{y_{1} y_{2}}\right) \exp \left(m \sqrt{-1}\left(\operatorname{Arg}\left(y_{1}+y_{2}+\sqrt{-1} t\right)+\phi_{1}-\phi^{\prime}\right)\right) d t+O(1) \\
& =\frac{1}{r_{i}} \sqrt{y_{1} y_{2}} \exp \left(-m \sqrt{-1}\left(\phi_{1}-\phi^{\prime}\right)\right) g\left(\log y_{1}-\log y_{2}\right)+O(1) .
\end{aligned}
$$

Thus the lemma is proved.

Now we can write down the Selberg trace formula

$$
\begin{array}{rl}
\sum_{j} & h\left(\sqrt{-1 / 4-\lambda_{j}}\right) \operatorname{tr}\left(T(\Gamma \alpha \Gamma) ; \lambda_{j}\right) \\
& =\frac{1}{2 \pi} \int_{\Gamma \backslash \tilde{\boldsymbol{H}}} \operatorname{tr}(K(z, \phi, z, \phi)-H(z, \phi, z, \phi)) d z d \phi .
\end{array}
$$

So far, the validity of (15) is restricted by the condition on $\Phi$. But we can relax the corresponding condition on $h(r)$ as:

(i) $h(r)$ is analytic in the region $|\operatorname{Im}(r)|<\max ((|m|-1) / 2,1 / 2))$

(ii) $h(r)=h(-r)$

(iii) $h(r) \leq A(1+|r|)^{-2-\varepsilon}$

where $A$ is some positive constant and $\varepsilon$ is an arbitrary positive number. This is because that $T(\Gamma \alpha \Gamma)$ is a bounded operator by the commensurability of $\Gamma$ and $\alpha \Gamma \alpha^{-1}$ and hence the eigenvalues of $T(\Gamma \alpha \Gamma)$ on $L_{x}(m, \lambda)$ are uniformly bounded with respect to $\lambda$.

The right hand side of (15) is evaluated by the method of the truncated fundamental domain (see Kubota [10]). We shall give the brief sketch of this classical method. Let $V_{i}$ be the neighborhood of $\kappa_{i}$ such that $\sigma_{i}^{-1} V_{i}=\{z \in \boldsymbol{H} \mid \operatorname{Im}(z)>Y\}, \boldsymbol{H}^{*}=\boldsymbol{H}-\cup_{i} \cup_{\sigma \in \Gamma} \sigma V_{i}$, and $\tilde{\boldsymbol{H}}^{*}=\boldsymbol{H}^{*} \times$ T. First we study two integrals

$$
\begin{aligned}
& \frac{1}{2 \pi} \int_{\Gamma \backslash \tilde{\boldsymbol{H}}^{*}} \operatorname{tr} K(z, \phi, z, \phi) d z d \phi \\
& \quad=\frac{1}{2 \pi} \sum_{[g]} \operatorname{tr} \chi(g) \int_{\Gamma(g) \backslash \tilde{\boldsymbol{H}}^{*}} k(z, \phi, g(z, \phi)) d z d \phi
\end{aligned}
$$

and

$$
\frac{1}{2 \pi} \int_{\Gamma \backslash \tilde{\boldsymbol{H}}^{*}} \operatorname{tr} H(z, \phi, z, \phi) d z d \phi
$$


Cancelling $\log Y$ term and letting $Y \rightarrow \infty$, we can get the right hand side of (15). To evaluate (16), we classify the conjugacy classes of $\Gamma \alpha \Gamma$ in five types:

( I ) Identity

(II) Elliptic conjugacy classes

(III) Hyperbolic conjugacy classes which fix hyperbolic points of $\Gamma$

(IV) Hyperbolic conjugacy classes which fix cusps of $\Gamma$

(V) Parabolic conjugacy classes

The explicit evaluations of these classes will be given in $\S 2-\S 6$.

\section{§ 2. Contributions from identity and elliptic conjugacy classes}

These parts were calculated by Hejhal in the case of trace formula for $\alpha=1$. See Hejhal [6], p. 389 p. 397. There is no change when we treat our case.

( I ) Identity

The contribution from the identity exists only when $\alpha \in \Gamma$ and is given by

$$
\begin{aligned}
J(\mathrm{id})= & \nu \operatorname{vol}(\Gamma \backslash \boldsymbol{H}) \Phi(0) \\
= & -\frac{\nu \operatorname{vol}(\Gamma \backslash \boldsymbol{H})}{2 \pi} \int_{-\infty}^{\infty} \frac{g^{\prime}(u) \exp (-m u / 2)}{\exp (u / 2)-\exp (-u / 2)} d u \\
= & \frac{\nu \operatorname{vol}(\Gamma \backslash \boldsymbol{H})}{4 \pi} \int_{-\infty}^{\infty} r h(r) \frac{\sinh (2 \pi r)}{\cosh (2 \pi r)+\cos (\pi m)} d r \\
& +\frac{\nu \operatorname{vol}(\Gamma \backslash \boldsymbol{H})}{4 \pi} \sum_{\substack{l: \text { odd } \\
1 \leq i \leq|m|}}(|m|-l) h\left[\sqrt{-1}\left(\frac{|m|-l}{2}\right)\right] .
\end{aligned}
$$

(II) Elliptic conjugacy classes

Let $R$ be a representative of an elliptic conjugacy class of $\Gamma \alpha \Gamma$. Then

$$
R \sim\left(\begin{array}{rr}
\cos \theta & -\sin \theta \\
\sin \theta & \cos \theta
\end{array}\right)
$$

where $\sim$ is the conjugation in $\mathrm{SL}_{2}(\boldsymbol{R})$. In the case that $\Gamma$ contains -1 , we may assume that $0<\theta<\pi$. But in our case, we can't assume this. First of all, we treat the case that $0<\theta<\pi$. Then the contribution is

$$
\begin{aligned}
& \frac{\sqrt{-1} \operatorname{tr} \chi(R) \exp (\sqrt{-1}(m-1) \theta)}{4 \# \Gamma(R) \sin \theta} \\
& \quad \times \int_{-\infty}^{\infty} g(u) \exp \left(\frac{(m-1) u}{2}\right) \frac{\exp u-\exp 2 \sqrt{-1} \theta}{\cosh u-\cos 2 \theta} d u .
\end{aligned}
$$


Using (14) to write this integral in terms of $h(r)$, we get; if $m=2 N+2$ then

$$
\begin{aligned}
I(R)= & \frac{\operatorname{tr} \chi(R)}{4 \# \Gamma(R) \sin \theta}\left(\int_{-\infty}^{\infty} h(r) \frac{\cosh 2 r(\pi-\theta)+\cosh 2 r \theta}{1+\cosh 2 \pi r} d r+\right. \\
& \left.\sum_{l=0}^{N} 2 \sqrt{-1} \exp (\sqrt{-1}(m-1-2 l) \theta) h\left[\sqrt{-1}\left(\frac{m-1-2 l}{2}\right)\right]\right),
\end{aligned}
$$

if $m=2 N+1$ then

$$
\begin{aligned}
I(R)= & \frac{\operatorname{tr} \chi(R)}{4 \# \Gamma(R) \sin \theta}\left(\int_{-\infty}^{\infty} h(r) \frac{\cosh 2 r(\pi-\theta)-\cosh 2 r \theta}{\cosh 2 \pi r-1} d r+\right. \\
& \left.-\sqrt{-1} h(0)+\sum_{l=0}^{N} 2 \sqrt{-1} \exp (2 \sqrt{-1}(N-l) \theta) h[\sqrt{-1}(N-l)]\right) .
\end{aligned}
$$

We can remove the assumption of the range of $\theta$, because the point pair invariant function

$$
\operatorname{Arg}\left(\frac{z-\overline{R z^{\prime}}}{2 \sqrt{-1}}\right)+\phi-\left(\phi^{\prime}+\operatorname{Arg} j\left(R, z^{\prime}\right)\right)
$$

changes its sign when we replace $R$ with $R^{-1}$ in (8).

\section{§3. Contribution from hyperbolic conjugacy classes which fix hyperbolic points of $\Gamma$}

Let $T$ be a hyperbolic element of $\Gamma \alpha \Gamma$ which fix hyperbolic points of $\Gamma$, such that

$$
T \sim\left(\begin{array}{cc}
\lambda & \\
& \lambda^{-1}
\end{array}\right) \text { and }|\lambda|>1
$$

Let $T_{0}$ be the generator of $\Gamma(T)$ and $\lambda_{0}$ is the eigenvalue of $T_{0}$ such that $\left|\lambda_{0}\right|>1$. Then the contribution of the hyperbolic conjugacy class represented by $T$ is

$$
\begin{aligned}
& I(T)=\int_{\Gamma^{\prime}(T) \backslash \boldsymbol{H}} \Phi\left(\frac{\left|z-\lambda^{2} z\right|^{2}}{\lambda^{2} y^{2}}\right) \sqrt{-1^{m}} \exp \left(-\sqrt{-1} m \operatorname{Arg}\left(z-\lambda^{2} \bar{z}\right)\right) \frac{\lambda^{-m}}{\left|\lambda^{-1}\right|^{m}} d z \\
& \quad=\sqrt{-1}^{m} \operatorname{sgn} \lambda^{m} \int_{-\infty}^{\infty} \int_{1}^{\lambda_{0}^{2}} \Phi\left(\frac{\left(\lambda^{2}-1\right)^{2}}{\lambda^{2}}\left(1+\frac{x^{2}}{y^{2}}\right)\right) \frac{\left|\left(1-\lambda^{2}\right) x+\left(1+\lambda^{2}\right) y \sqrt{-1}\right|^{m}}{\left\{\left(1-\lambda^{2}\right) x+\left(1+\lambda^{2}\right) y \sqrt{-1}\right\}^{m}} \frac{d x d y}{y^{2}} .
\end{aligned}
$$

Substituting $x$ by $\left(|\lambda| y /\left(\lambda^{2}-1\right)\right) x$ and letting $\omega=\left(\lambda^{2}-1\right)^{2} / \lambda^{2}$, we get

$$
=\operatorname{sgn} \lambda^{m} \log \lambda_{0}^{2} \int_{-\infty}^{\infty} \Phi\left(x^{2}+\omega\right) \frac{|\sqrt{\omega+4}+x \sqrt{-1}|^{m}}{\{\sqrt{\omega+4}+x \sqrt{-1}\}^{m}} \frac{|\lambda|}{\lambda^{2}-1} d x
$$




$$
=\operatorname{sgn} \lambda^{m} \log \lambda_{0}^{2} \frac{|\lambda| g\left(\log \lambda^{2}\right)}{\lambda^{2}-1} .
$$

\section{$\S 4$. Contributions from hyperbolic conjugacy classes which fix cusps of $\Gamma$}

Let $T$ be a representative of a hyperbolic conjugacy class of $\Gamma \alpha \Gamma$ which fixes a cusp of $\Gamma$. If one of the fixed points of $T$ is a cusp of $\Gamma$, then the other one is also a cusp of $\Gamma$. In this case, we can see that $\Gamma(T)=\{1\}$.

For simplicity, we assume that cusps, say $\kappa_{1}$ and $\kappa_{2}$, are the fixed points of $T$. Let $\varphi$ be an element of $G$ such that $\varphi\left(\kappa_{1}\right)=\infty, \varphi\left(\kappa_{2}\right)=0$ and

$$
\varphi T_{\varphi}^{-1}=\left(\begin{array}{ll}
\lambda & \\
& \lambda^{-1}
\end{array}\right)
$$

where $|\lambda|>1$. Then the contribution is

$$
\sqrt{-1}^{m} \operatorname{sgn} \lambda^{m} \int_{\varphi\left(\boldsymbol{H}^{*}\right)} \Phi\left(\frac{\left(\lambda^{2}-1\right)^{2}}{\lambda^{2}}\left(1+\frac{x^{2}}{y^{2}}\right)\right) \frac{\left|\left(1-\lambda^{2}\right) x+\left(1+\lambda^{2}\right) y \sqrt{-1}\right|^{m}}{\left\{\left(1-\lambda^{2}\right) x+\left(1+\lambda^{2}\right) y \sqrt{-1}\right\}^{m}} \frac{d x d y}{y^{2}},
$$

where $\boldsymbol{H}^{*}$ is defined in $\S 1$. We can neglect the integral over $\varphi \sigma V_{i}$ $(i \neq 1,2)$, because it converges to zero when $Y$ approaches to infinity. So we can change the domain of integral $\varphi\left(\boldsymbol{H}^{*}\right)$ with $\boldsymbol{H}_{1}=\boldsymbol{H}-(\boldsymbol{A} \cup B)$, where $A$ (resp. $B$ ) is the neighborhood of $\infty$ (resp. 0 ). We may choose $\varphi$ so that the above properties hold and

$$
\begin{aligned}
& A=\varphi V_{1}=\{z \in \boldsymbol{H} \mid \operatorname{Im}(z)>Y\}, \\
& B=\varphi V_{2}=\tau\{z \in \boldsymbol{H} \mid \operatorname{Im}(z)>Y\},
\end{aligned}
$$

where $\varphi \sigma_{1}=\left(\begin{array}{ll}1 & * \\ 0 & *\end{array}\right), \tau \in G$, and $\tau(\infty)=0$, because we can replace $\varphi$ by $\left(\begin{array}{ll}a & \\ & a^{-1}\end{array}\right) \varphi$, where $a \in \boldsymbol{R}$.

Let $B$ be the circle which passes 0 and is tangent to the real axis. Denote by $c(\tau)$ the $(2,1)$ element of $\tau$, which depends only on $\kappa_{1}$ and $\kappa_{2}$. The radius of $B$ is $1 /\left(2 c(\tau)^{2} Y\right)$, which we denote by $\rho$. According to these notations, the contribution is

$\sqrt{-1}^{m} \operatorname{sgn} \lambda^{m} \int_{\boldsymbol{H}_{\mathbf{1}}} \Phi\left(\frac{\left(\lambda^{2}-1\right)^{2}}{\lambda^{2}}\left(1+\frac{x^{2}}{y^{2}}\right)\right) \frac{\left|\left(1-\lambda^{2}\right) x+\left(1+\lambda^{2}\right) y \sqrt{-1}\right|^{m}}{\left\{\left(1-\lambda^{2}\right) x+\left(1+\lambda^{2}\right) y \sqrt{-1}\right\}^{m}} \frac{d x d y}{y^{2}}+o(1)$

where $o$ is the Landau symbol when $Y \rightarrow \infty$. From now on in this section, we neglect $o(1)$. Changing variables, we get 


$$
\begin{aligned}
\sqrt{-1}^{m} & \operatorname{sgn} \lambda^{m} \int_{0}^{\pi} \int_{2 \rho \sin \theta}^{Y / \sin \theta} \Phi\left(\frac{\left(\lambda^{2}-1\right)^{2}}{\lambda^{2}}\left(1+\cot ^{2} \theta\right)\right) \\
& \times \frac{\left|\left(1-\lambda^{2}\right) \cot \theta+\left(1+\lambda^{2}\right) \sqrt{-1}\right|^{m}}{\left\{\left(1-\lambda^{2}\right) \cot \theta+\left(1+\lambda^{2}\right) \sqrt{-1}\right\}^{m}} \frac{r d r d \theta}{r^{2} \sin ^{2} \theta} \\
= & \sqrt{-1^{m}} \operatorname{sgn} \lambda^{m} \int_{-\infty}^{\infty} \Phi\left(\frac{\left(\lambda^{2}-1\right)^{2}}{\lambda^{2}}\left(1+t^{2}\right)\right) \frac{\mid\left(1-\lambda^{2}\right) t+\left(1+\lambda^{2}\right) \sqrt{-1}}{\left\{\left(1-\lambda^{2}\right) t+\left(1+\lambda^{2}\right) \sqrt{-1}\right\}^{m}} \\
& \times\left(2 \log Y|c(\tau)|+\log \left(1+t^{2}\right)\right) d t .
\end{aligned}
$$

Let $\omega=\left(1-\lambda^{2}\right)^{2} / \lambda^{2}$, and replace $t$ by $\left(|\lambda| /\left(\lambda^{2}-1\right)\right) t$. Then

$$
\begin{aligned}
= & \operatorname{sgn} \lambda^{m}(2 \log Y|c(\tau)|-\log \omega) \frac{|\lambda|}{\lambda^{2}-1} \int_{-\infty}^{\infty} \Phi\left(\omega+t^{2}\right) \frac{|\sqrt{\omega+4}+t \sqrt{-1}|^{m}}{\{\sqrt{\omega+4}+t \sqrt{-1}\}^{m}} d t \\
& +\frac{|\lambda| \operatorname{sgn} \lambda^{m}}{\lambda^{2}-1} \int_{-\infty}^{\infty} \log \left(\omega+t^{2}\right) \Phi\left(\omega+t^{2}\right) \frac{|\sqrt{\omega+4}+t \sqrt{-1}|^{m}}{\{\sqrt{\omega+4}+t \sqrt{-1}\}^{m}} d t \\
= & \frac{2|\lambda| \operatorname{sgn} \lambda^{m}}{\lambda^{2}-1} \log \left(\frac{Y|c(\tau) \lambda|}{\lambda^{2}-1}\right) Q(\omega) \\
& +\frac{|\lambda| \operatorname{sgn} \lambda^{m}}{\lambda^{2}-1} \int_{-\infty}^{\infty} \log \left(\omega+t^{2}\right) \Phi\left(\omega+t^{2}\right) \frac{|\sqrt{\omega+4}+t \sqrt{-1}|^{m}}{\{\sqrt{\omega+4}+t \sqrt{-1}\}^{m}} d t .
\end{aligned}
$$

For the last integral, we need more calculation.

$$
\begin{aligned}
\int_{-\infty}^{\infty} \log \left(\omega+t^{2}\right) \Phi\left(\omega+t^{2}\right) \frac{|\sqrt{\omega+4}+t \sqrt{-1}|^{m}}{\{\sqrt{\omega+4}+t \sqrt{-1}\}^{m}} d t \\
=\int_{\omega}^{\infty} \log (u) \Phi(u) \operatorname{Re}\left[\left(\frac{\sqrt{\omega+4}+\sqrt{u-\omega} \sqrt{-1}}{\sqrt{\omega+4}-\sqrt{u-\omega} \sqrt{-1}}\right)^{m / 2}\right] \frac{d u}{\sqrt{u-\omega}} \\
=-\frac{1}{\pi} \int_{-\infty}^{\infty} \int_{\omega+\xi^{2}}^{\infty} \frac{\log \left(u-\xi^{2}\right)}{\sqrt{u-\xi^{2}-\omega} Q^{\prime}(u)} \\
\quad \times\left[\frac{\sqrt{u+4}-\xi}{\sqrt{u+4}+\xi}\right]^{m / 2} \operatorname{Re}\left[\left(\frac{\sqrt{\omega+4}+\sqrt{u-\xi^{2}-\omega} \sqrt{-1}}{\sqrt{\omega+4}-\sqrt{u-\xi^{2}-\omega} \sqrt{-1}}\right)^{m / 2}\right] d u d \xi . \\
=-\frac{1}{\pi} \int_{0}^{\infty} Q^{\prime}(u+\omega) J(u) d u
\end{aligned}
$$

where

$$
J(u)=\operatorname{Re} \int_{-\pi / 2}^{\pi / 2} \log \left(\omega+u \cos ^{2} \theta\right)\left(\frac{\sqrt{\omega+4}+\sqrt{-u} \cos \theta}{\sqrt{u+\omega+4}+\sqrt{u} \cos \theta}\right)^{m} d \theta .
$$

Then

$$
J(u)=\operatorname{Re} \int_{-\pi / 2}^{\pi / 2} \log \left(\omega+u \cos ^{2} \theta\right)\left(\frac{\sinh \gamma+\sqrt{-1} \cos \theta}{\cosh \gamma+\sin \theta}\right)^{m} d \theta,
$$

where $\gamma$ is the value which satisfies 


$$
\sinh \gamma=\frac{\sqrt{\omega+4}}{\sqrt{u}}, \quad \cosh \gamma=\frac{\sqrt{u+\omega+4}}{\sqrt{u}} .
$$

Let $C_{1}$ be the semicircular path from $-\sqrt{-1}$ to $\sqrt{-1}$ which encircles the origin anticlockwisely. $C_{2}, C_{4}, C_{6}, C_{8}$ is the line segment from $-\sqrt{-1}$ to $-(a+\varepsilon) \sqrt{-1},-(a-\varepsilon) \sqrt{-1}$ to $-\varepsilon \sqrt{-1}, \varepsilon \sqrt{-1}$ to $(a-\varepsilon) \sqrt{-1}$, and $(a+\varepsilon) \sqrt{-1}$ to $\sqrt{-1}$, where $a=\sqrt{\omega / u+1}-\sqrt{\omega / u}$, and $\varepsilon$ is a sufficiently small positive number. $C_{3}$ (resp. $C_{7}$ ) is the anticlockwise semicircular path from $-(a+\varepsilon) \sqrt{-1}$ to $-(a-\varepsilon) \sqrt{-1}$ (resp. $(a-\varepsilon) \sqrt{-1}$ to $(a+\varepsilon) \sqrt{-1})$ whose center is $-a \sqrt{-1}$ (resp. $a \sqrt{-1}$ ). $\quad C_{5}$ is the anticlockwise semicircular path from $-\varepsilon \sqrt{-1}$ to $\varepsilon \sqrt{-1}$. We see that

$$
\frac{\sinh \gamma+\sqrt{-1} \cos \theta}{\cosh \gamma+\sin \theta}=\frac{\sqrt{-1} e^{\gamma}-e^{\sqrt{-1} \theta}}{\sqrt{-1} e^{\gamma}+e^{\sqrt{-1} \theta}} \text {. }
$$

Thus

$$
J(u)=\operatorname{Re} \int_{C_{1}} \log \left[\omega+u\left(\frac{z+z^{-1}}{2}\right)^{2}\right]\left[\frac{\sqrt{-1} e^{\gamma}-z}{\sqrt{-1} e^{\gamma}+z}\right]^{m} \frac{d z}{\sqrt{-1} z} .
$$

The integrals of the same function over $C_{3}, C_{7}$ tend to 0 , when $\varepsilon \rightarrow 0$. Hence by Cauchy's theorem, we get

$$
\begin{aligned}
\int_{C_{1}} \log & {\left[\omega+u\left(\frac{z+z^{-1}}{2}\right)^{2}\right]\left[\frac{\sqrt{-1} e^{\gamma}-z}{\sqrt{-1} e^{\gamma}+z}\right]^{m} \frac{d z}{\sqrt{-1} z} } \\
= & \int_{C_{2}}+\int_{C_{4}}+\int_{C_{5}}+\int_{C_{8}}+\int_{C_{8}}+o(1) \\
= & \int_{C_{2}} \log \left[\omega+u\left(\frac{z+z^{-1}}{2}\right)^{2}\right]\left[\frac{\sqrt{-1} e^{\gamma}-z}{\sqrt{-1} e^{\gamma}+z}\right]^{m} \frac{d z}{\sqrt{-1} z} \\
& +\int_{C_{4}}\left(\log \left[\omega+u\left(\frac{z+z^{-1}}{2}\right)^{2}\right]+\pi \sqrt{-1}\right)\left[\frac{\sqrt{-1} e^{\gamma}-z}{\sqrt{-1} e^{\gamma}+z}\right]^{m} \frac{d z}{\sqrt{-1} z} \\
& +\int_{C_{5}}\left(\log \left[\omega+u\left(\frac{z+z^{-1}}{2}\right)^{2}\right]+\pi \sqrt{-1}\right)\left[\frac{\sqrt{-1} e^{\gamma}-z}{\sqrt{-1} e^{\gamma}+z}\right]^{m} \frac{d z}{\sqrt{-1} z} \\
& +\int_{C_{6}}\left(\log \left[\omega+u\left(\frac{z+z^{-1}}{2}\right)^{2}\right]-\pi \sqrt{-1}\right)\left[\frac{\sqrt{-1} e^{\gamma}-z}{\sqrt{-1} e^{\gamma}+z}\right]^{m} \frac{d z}{\sqrt{-1} z} \\
& +\int_{C_{8}} \log \left[\omega+u\left(\frac{z+z^{-1}}{2}\right)^{2}\right]\left[\frac{\sqrt{-1} e^{\gamma}-z}{\sqrt{-1} e^{\gamma}+z}\right]^{m} \frac{d z}{\sqrt{-1} z}+o(1),
\end{aligned}
$$

where $o(1)$ is the Landau symbol when $\varepsilon \rightarrow 0$. In the above formula, the branch of the logarithm in the region enclosed by $C_{i}(i=1,2, \cdots, 8)$ is taken so that the value on $C_{1}$ is real. Considering real parts of both sides, we see 


$$
\begin{aligned}
J(u)= & \pi \operatorname{Re} \int_{C_{4}}\left[\frac{\sqrt{-1} e^{\gamma}-z}{\sqrt{-1} e^{\gamma}+z}\right]^{m} \frac{d z}{z} \\
& +\operatorname{Re} \int_{C_{5}}\left(\log \left[\omega+u\left(\frac{z+z^{-1}}{2}\right)^{2}\right]+\pi \sqrt{-1}\right)\left[\frac{\sqrt{-1} e^{\gamma}-z}{\sqrt{-1} e^{\gamma}+z}\right]^{m} \frac{d z}{\sqrt{-1} z} \\
& -\pi \operatorname{Re} \int_{C_{6}}\left[\frac{\sqrt{-1} e^{\gamma}-z}{\sqrt{-1} e^{\gamma}+z}\right]^{m} \frac{d z}{z}+o(1) .
\end{aligned}
$$

On the path $C_{5}$, we have

$$
\left[\frac{\sqrt{-1} e^{r}-z}{\sqrt{-1} e^{r}+z}\right]^{m}=1+o(\varepsilon)
$$

as $\varepsilon \rightarrow 0$. Thus we can deduce that

$$
\begin{aligned}
J(u)= & \pi \operatorname{Re} \int_{-a}^{-\varepsilon}\left[\frac{e^{\gamma}-y}{e^{\gamma}+y}\right]^{m} \frac{d y}{y}-\pi \operatorname{Re} \int_{\varepsilon}^{a}\left[\frac{e^{\gamma}-y}{e^{\gamma}+y}\right]^{m} \frac{d y}{y} \\
& +\operatorname{Re} \int_{C_{5}} \log \left[\omega+u\left(\frac{z+z^{-1}}{2}\right)^{2}\right]\left[\frac{\sqrt{-1} e^{\gamma}-z}{\sqrt{-1} e^{\gamma}+z}\right]^{m} \frac{d z}{\sqrt{-1} z}+o(1) .
\end{aligned}
$$

From the estimation

$\log \left[\omega+u\left(\frac{\varepsilon e^{\sqrt{-1} \theta}+\varepsilon^{-1} e^{-\sqrt{-1} \theta}}{2}\right)^{2}\right]=\log u-\log 4-2 \log \varepsilon-2 \sqrt{-1} \theta+o\left(\varepsilon^{2}\right)$,

we get

$$
\begin{aligned}
J(u)= & -\pi \operatorname{Re} \int_{0}^{a}\left[\left(\frac{e^{\gamma}+y}{e^{\gamma}-y}\right)^{m}-1\right] \frac{d y}{y}-\pi \operatorname{Re} \int_{0}^{a}\left[\left(\frac{e^{\gamma}-y}{e^{\gamma}+y}\right)^{m}-1\right] \frac{d y}{y} \\
& +\pi(\log u-\log 4-2 \log a) .
\end{aligned}
$$

By the straight forward calculation, we get

$$
J^{\prime}(u)=\frac{\pi \sqrt{\omega+4}}{\sqrt{u+\omega+4} \sqrt{u+\omega}(\sqrt{u+\omega}+\sqrt{\omega})} .
$$

The contribution from this conjugacy class is given by,

$$
\frac{2|\lambda| \operatorname{sgn} \lambda^{m}}{\lambda^{2}-1} \log \left(\frac{Y|c(\tau) \lambda|}{\lambda^{2}-1}\right) Q(\omega)-\frac{|\lambda| \operatorname{sgn} \lambda^{m}}{\pi\left(\lambda^{2}-1\right)} \int_{0}^{\infty} Q^{\prime}(\omega+u) J(u) d u \text {. }
$$

We will see that the $\log Y$ terms cancel out with those of (17) in $\S 6$. Using (20), we have the result.

TheOREM 1. Let $T$ be a hyperbolic element of $\Gamma \alpha \Gamma$ which fixes cusps of $\Gamma$, and $\lambda, \lambda^{-1}$ be its eigen values where $|\lambda|>1$. Then the contribution 
from the conjugacy class of $T$ is given by

$$
\begin{aligned}
& \frac{2|\lambda| \operatorname{sgn} \lambda^{m}}{\lambda^{2}-1}[\log (|c(\tau)|) g(2 \log |\lambda|) \\
& \quad-\int_{2 \log |\lambda|}^{\infty} g(u)\left[\left(e^{m u / 2}|\lambda|^{-m}+e^{-m u / 2}|\lambda|^{m}-2\right) \frac{e^{u / 2}|\lambda|-1}{e^{u / 2}-|\lambda|}\right. \\
& \left.\left.\quad-e^{u / 2}-e^{-u / 2}\right] \frac{d u}{e^{u / 2}-e^{-u / 2}+|\lambda|-|\lambda|^{-1}}\right]
\end{aligned}
$$

where $c(\tau)$ is defined as above.

\section{§5. Contribution from parabolic conjugacy classes}

Put $B_{i}=\left\{g \in \Gamma \alpha \Gamma\left|g \kappa_{i}=\kappa_{i},\right| \operatorname{tr} g \mid=2\right\}$, then

$$
B_{i}=\bigcup_{\mu} \alpha_{\mu} \Gamma_{i} \text { (finite disjoint union). }
$$

Each element $g \in B_{i}$ can be written in the form

$$
g=s(g) \sigma_{i}\left(\begin{array}{cc}
1 & v(g) \\
0 & 1
\end{array}\right) \sigma_{i}^{-1}
$$

where $s(g)= \pm 1, v(g) \in \boldsymbol{R}$. We may assume that $v\left(\alpha_{\mu}\right) \in[0,1)$. We consider the sum

$$
\frac{1}{2 \pi} \sum_{g \in \alpha_{\mu} \Gamma_{i}}^{\prime} \operatorname{tr} \chi(g) \int_{\Gamma_{i} \backslash \mathscr{H}^{*}} k(z, \phi, g(z, \phi)) d z d \phi
$$

where $\sum^{\prime}$ is the summation which avoids $g=1$. Put

$$
\begin{aligned}
& \chi\left(\alpha_{\mu}\right) s\left(\alpha_{\mu}\right)^{m} \sim\left(\begin{array}{lll}
\exp \left(2 \pi \sqrt{-1} \beta_{1 \mu}\right) & \\
& \cdot & \\
& & \exp \left(2 \pi \sqrt{-1} \beta_{\nu \mu}\right)
\end{array}\right), \\
& \chi(\eta) s(\eta)^{m} \sim\left(\begin{array}{lll}
\exp \left(2 \pi \sqrt{-1} \zeta_{1}\right) & & \\
& \cdot & \\
& & \exp \left(2 \pi \sqrt{-1} \zeta_{\nu}\right)
\end{array}\right),
\end{aligned}
$$

where $\eta$ is a generator of $\Gamma_{i}$. Then

$$
\begin{aligned}
(21)= & \sum_{j=1}^{\nu} \lim _{Y \rightarrow \infty}\left[\int_{0}^{Y} \int_{0}^{1} \sum_{n \in Z}^{\prime} \exp \left(2 \pi \sqrt{-1}\left(n \zeta_{j}+\beta_{j \mu}\right)\right)\right. \\
& \left.\times \Phi\left(\frac{\left\{n+v\left(\alpha_{\mu}\right)\right\}^{2}}{y^{2}}\right) e^{-\sqrt{-1} m \operatorname{Arg}\left\{2 y+\sqrt{-1}\left(n+v\left(\alpha_{\mu}\right)\right)\right\}} \frac{d x d y}{y^{2}}\right] .
\end{aligned}
$$

So we study the next integral 


$$
\begin{aligned}
I(\zeta, v) & =\int_{0}^{Y} \sum_{n \in Z}^{\prime} \exp (2 \pi \sqrt{-1} n \zeta) \Phi\left(\frac{(n+v)^{2}}{y^{2}}\right) e^{-\sqrt{-1} m \operatorname{Arg}\{2 y+\sqrt{-1}(n+v)\}} \frac{d y}{y^{2}} \\
& =\sum_{n \in Z}^{\prime} \int_{(n+v) \infty}^{(n+v) / Y} \exp (2 \pi \sqrt{-1} n \zeta) \Phi\left(u^{2}\right) e^{-\sqrt{-1} m \operatorname{Arg}(2+\sqrt{-1} u)} \frac{d u}{-n-v}
\end{aligned}
$$

As $\zeta$ can be written in the form $t / r_{i}\left(t=0,1, \cdots, r_{i}-1\right)$, we replace $n$ by $n r_{i}+k\left(k=0,1, \cdots, r_{i}\right)$. Then

$$
I(\zeta, v)=\sum_{k=0}^{r_{i}-1} \exp \left(2 \pi \sqrt{-1} \frac{k t}{r_{i}}\right) \sum_{n \in Z}^{*} \int_{\left(n r_{i}+k+v\right) \infty}^{\left(n r_{i}+k+v\right) / Y} \Phi\left(u^{2}\right) e^{-\sqrt{-1} m \operatorname{Arg}(2+\sqrt{-1} u)} \frac{d u}{-n r_{i}-k-v},
$$

where $\sum^{*}$ runs over all $n \in Z$ except $n=k=v=0$. We put

$$
\begin{aligned}
& J(d)=\sum_{n=1}^{\infty} \int_{\left(n r_{i}+d\right) / Y}^{\infty} \xi(u) \frac{d u}{n r_{i}+d}, \\
& \xi(u)=\Phi\left(u^{2}\right) e^{-\sqrt{-1} m \operatorname{Arg}(2+\sqrt{-1} u)} .
\end{aligned}
$$

Then we can see that

$$
I(\zeta, v)=\sum_{k=0}^{r_{i}-1} \exp (2 \pi \sqrt{-1} k \zeta)\left(J(k+v)+\overline{J(-k-v)}+\delta(k=v=0) \int_{(k+v) / Y}^{\infty} \xi(u) d u\right),
$$

where

$$
\delta=\delta(k=v=0)=\left\{\begin{array}{ll}
0 & \text { if } k=v=0 \\
1 & \text { otherwise }
\end{array} .\right.
$$

LEMma 3. We have

$$
\begin{aligned}
\sum_{n=1}^{\infty} \frac{r}{A+r n} \int_{(A+r n) / Y}^{\infty} \xi(u) d u \\
\quad=\left(-\frac{\Gamma^{\prime}}{\Gamma}(1+A / r)+\log (Y / r)\right) \int_{0}^{\infty} \xi(u) d u+\int_{0}^{\infty} \xi(u) \log (u) d u+o(1)
\end{aligned}
$$

as $Y \rightarrow \infty$.

To prove this, we use the Euler-Maclaurin summation formula. (See Kubota [10], p. 103 104). Using this lemma, we get

$$
\begin{aligned}
J(k+v)= & \frac{1}{r_{i}}\left[\left(-\frac{\Gamma^{\prime}}{\Gamma}\left(1+\frac{k+v}{r_{i}}\right)+\log \left(\frac{Y}{r_{i}}\right)\right) \int_{0}^{\infty} \xi(u) d u+\int_{0}^{\infty} \xi(u) \log (u) d u\right] \\
& +o(1) .
\end{aligned}
$$

Hence we see

$$
I(\zeta, v)=\sum_{k=0}^{r_{i}-1} \frac{e^{2 \pi \sqrt{-1} k \zeta}}{r_{i}}\left[2 \operatorname{Re} \int_{0}^{\infty} \xi(u) \log (u) d u\right.
$$




$$
\begin{aligned}
& +\left(-\frac{\Gamma^{\prime}}{\Gamma}\left(1+\frac{k+v}{r_{i}}\right)-\frac{\Gamma^{\prime}}{\Gamma}\left(1-\frac{k+v}{r_{i}}\right)+\frac{\delta r_{i}}{k+v}+2 \log \frac{Y}{r_{i}}\right) \operatorname{Re} \int_{0}^{\infty} \xi(u) d u \\
& \left.+\sqrt{-1}\left(-\frac{\Gamma^{\prime}}{\Gamma}\left(1+\frac{k+v}{r_{i}}\right)+\frac{\Gamma^{\prime}}{\Gamma}\left(1-\frac{k+v}{r_{i}}\right)+\frac{\delta r_{i}}{k+v}\right) \operatorname{Im} \int_{0}^{\infty} \xi(u) d u\right]
\end{aligned}
$$

By the property of digamma function, we have

$$
\pi \cot ^{*}\left(\frac{\pi(k+v)}{r_{i}}\right)=-\frac{\Gamma^{\prime}}{\Gamma}\left(1+\frac{k+v}{r_{i}}\right)+\frac{\Gamma^{\prime}}{\Gamma}\left(1-\frac{k+v}{r_{i}}\right)+\frac{\delta r_{i}}{k+v}
$$

where

$$
\cot ^{*}(x)=\left\{\begin{array}{ll}
\cot (x) & \text { for } x \neq 0(\bmod \pi) \\
0 & \text { for } x \equiv 0(\bmod \pi)
\end{array} .\right.
$$

LEMMA 4. We have

$$
\begin{aligned}
& \int_{0}^{\infty} \Phi\left(u^{2}\right) e^{-m \sqrt{-1} \operatorname{Arg}(2+\sqrt{-1} u)} d u \\
& \quad=\frac{1}{2} g(0)-\frac{\sqrt{-1}}{4 \pi} \int_{-\infty}^{\infty} g(u) \exp \left(\frac{m-1}{2} u\right)\left(\frac{e^{u}-1}{\cosh u-1}\right) d u .
\end{aligned}
$$

LEMMA 5. We have

$$
\begin{aligned}
\operatorname{Re} \int_{0}^{\infty} \Phi\left(u^{2}\right) \log (u) e^{-m \sqrt{-1} \operatorname{Arg}(2+\sqrt{-1} u)} d u \\
=(-2 \gamma-\log 4) g(0)+h(0) / 2-\frac{1}{\pi} \int_{-\infty}^{\infty} h(r) \frac{\Gamma^{\prime}}{\Gamma}(1+\sqrt{-1} r) d r \\
\quad+\int_{0}^{\infty} \frac{g(u)}{\sinh (u / 2)}\left(1-\cosh \left(\frac{m u}{2}\right)\right) d u
\end{aligned}
$$

where $\gamma$ is the Euler constant.

For the proof of these lemmas, see Hejhal [6] p. 399 p. 400, p. 407 p. 411.

Using these facts, we have

$$
\begin{aligned}
I(\zeta, v) & =\sum_{k=0}^{r_{i}-1} \frac{e^{2 \pi \sqrt{-1} k \zeta}}{2 r_{i}}\left[\int_{0}^{\infty} \frac{g(u)}{\sinh (u / 2)}\left(1-\cosh \left(\frac{m u}{2}\right)\right) d u\right. \\
+ & (-2 \gamma-\log 4) g(0)+h(0) / 2-\frac{1}{\pi} \int_{-\infty}^{\infty} h(r) \frac{\Gamma^{\prime}}{\Gamma}(1+\sqrt{-1} r) d r \\
& +\left(-\frac{\Gamma^{\prime}}{\Gamma}\left(1+\frac{k+v}{r_{i}}\right)-\frac{\Gamma^{\prime}}{\Gamma}\left(1-\frac{k+v}{r_{i}}\right)+\frac{\delta r_{i}}{k+v}+2 \log \frac{Y}{r_{i}}\right) g(0) \\
& \left.-\frac{\sqrt{-1}}{2} \cot ^{*}\left(\pi \frac{(k+v)}{r_{i}}\right) \int_{-\infty}^{\infty} g(u) \exp \left(\frac{m-1}{2} u\right)\left(\frac{e^{u}-1}{\cosh u-1}\right) d u\right]+o(1) .
\end{aligned}
$$


As the $\log Y$ term will cancel out with that of (16) in $\S 6$, we have

THEOREM 2. The notation being as above, the contribution from parabolic conjugacy classes of $\Gamma \alpha \Gamma$ is given by

$$
\begin{aligned}
\sum_{i=1}^{\infty} \sum_{\mu} & \sum_{j=1}^{\nu} \exp \left(2 \pi \sqrt{-1} \beta_{j \mu}\right) I\left(\zeta_{j}, v\left(\alpha_{\mu}\right)\right)-(\log Y \text { term }) \\
= & \sum_{i=1}^{\infty} \sum_{\mu} \sum_{j=1}^{\nu} \exp \left(2 \pi \sqrt{-1} \beta_{j \mu}\right)\left[-\left\{\gamma+\log 2+\frac{1}{2}\left(\frac{\Gamma^{\prime}}{\Gamma}\left(1+v\left(\alpha_{\mu}\right)\right)\right.\right.\right. \\
& \left.\left.+\frac{\Gamma^{\prime}}{\Gamma}\left(1-v\left(\alpha_{\mu}\right)\right)-\frac{\left.\delta\left(v\left(\alpha_{\mu}\right)=0\right)\right)}{v\left(\alpha_{\mu}\right)}\right)\right\} g(0)+\frac{h(0)}{4} \\
& -\frac{1}{2 \pi} \int_{-\infty}^{\infty} h(r) \frac{\Gamma^{\prime}}{\Gamma}(1+\sqrt{-1} r) d r \\
& -\frac{\sqrt{-1}}{4} \cot ^{*}\left(\pi v\left(\alpha_{\mu}\right)\right) \int_{-\infty}^{\infty} g(u) \exp \left(\frac{m-1}{2} u\right)\left(\frac{e^{u}-1}{\cosh u-1}\right) d u \\
& \left.+\frac{1}{2} \int_{0}^{\infty} \frac{g(u)}{\sinh (u / 2)}\left(1-\cosh \left(\frac{m u}{2}\right)\right) d u\right] \\
& +\sum_{i=1}^{\infty} \sum_{\mu} \sum_{j=1}^{\nu} \sum_{k=0}^{r_{i}-1} \frac{e^{2 \pi} \sqrt{-1}\left(\beta_{j \mu}+\zeta_{j}\right) k}{2 r_{i}}\left[\left(-\frac{\Gamma^{\prime}}{\Gamma}\left(1+\frac{k+v\left(\alpha_{\mu}\right)}{r_{i}}\right)\right.\right. \\
& \left.-\frac{\Gamma^{\prime}}{\Gamma}\left(1-\frac{k+v\left(\alpha_{\mu}\right)}{r_{i}}\right)+\frac{\delta\left(k=v\left(\alpha_{\mu}\right)=0\right) r_{i}}{k+v\left(\alpha_{\mu}\right)}\right) g(0) \\
& \left.-\frac{\sqrt{-1}}{2} \cot ^{*}\left(\pi \frac{k+v\left(\alpha_{\mu}\right)}{r_{i}}\right) \int_{-\infty}^{\infty} g(u) \exp \left(\frac{m-1}{2} u\right)\left(\frac{e^{u}-1}{\cosh u-1}\right) d u\right],
\end{aligned}
$$

where

$$
\delta(\cdot)= \begin{cases}0 & \text { when the inner statement is true } \\ 1 & \text { otherwise. }\end{cases}
$$

Proof. Classify the summation according to $\zeta_{i}=0$ or $\zeta_{i} \neq 0$. If $\zeta_{i}$ $=0$ then we may replace $r_{i}$ with 1 throughout our arguments.

Remark. This result agrees with the parabolic part of Theorem 6.3 of Hejhal [6] p. 412 in the case that $\alpha \in \Gamma$. To see this, we should notice

$$
\sum_{k=1}^{r-1} \cos (2 \pi w k / r) \frac{\Gamma^{\prime}}{\Gamma}(k / r)=r+r \log (2 \sin (\pi w / r))
$$

and

$$
\sum_{k=1}^{r-1} \sin (2 \pi w k / r) \frac{\Gamma^{\prime}}{\Gamma}(k / r)=\pi(w-r / 2)
$$




\section{§6. Contribution from $H\left(z, \phi, z^{\prime}, \phi^{\prime}\right)$}

Finally, we will calculate the integral (17). To do this, we must know the constant term of the Fourier-Bessel expansion of $E_{i}(z, \phi ; s)$ and $F_{i}(z, \phi ; s)$ where

$$
F_{i}(z, \phi ; s)=\sum_{\mu} \chi\left(\alpha_{\mu}\right) E_{i}\left(\alpha_{\mu}^{-1}(z, \phi) ; s\right) .
$$

Denote $\Gamma_{\infty, i}=\sigma_{i}^{-1} \Gamma_{i} \sigma_{i}$. This definition depends only on the regularity of $\kappa_{i}$. Then $E_{i}\left(\sigma_{j}(z, \phi) ; s\right)$ and $F_{i}\left(\sigma_{j}(z, \phi) ; s\right)$ is invariant under the motion $z \rightarrow z+2 r_{i}$. (We must consider the case that $m$ is odd and $\kappa_{i}$ is irregular). Put

$$
\begin{aligned}
& E_{i}\left(\sigma_{j}(z, \phi) ; s\right)=E_{i j}(z, \phi ; s) \exp (-m \sqrt{-1} \phi), \\
& F_{i}\left(\sigma_{j}(z, \phi) ; s\right)=F_{i j}(z, \phi ; s) \exp (-m \sqrt{-1} \phi) .
\end{aligned}
$$

Then we can expand

$$
\begin{aligned}
& E_{i j}(z, \phi ; s)=\sum_{n \in Z} a_{n}(y, s) \exp \left(\frac{2 \pi \sqrt{-1} n x}{2 r_{i}}\right), \\
& F_{i j}(z, \phi ; s)=\sum_{n \in Z} b_{n}(y, s) \exp \left(\frac{2 \pi \sqrt{-1} n x}{2 r_{i}}\right) .
\end{aligned}
$$

LEMma 6. We have

$$
a_{0}(y, s)=\delta_{i j} y^{s} P_{i}+\varphi_{i j}(s) y^{1-s}
$$

where

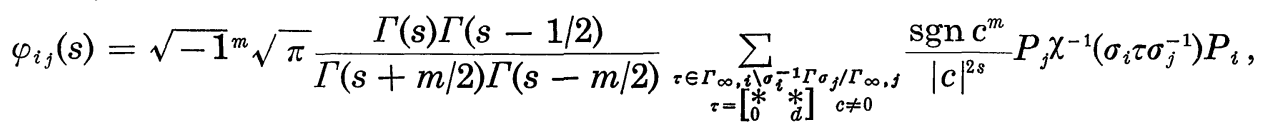

and $b_{0}(y, s)=w_{i j}(s) y^{s}+\varphi_{i j}^{*}(s) y^{1-s}$,

where

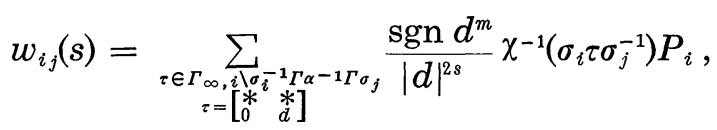

and

$$
\begin{aligned}
& \varphi_{i j}^{*}(s)=\sqrt{-1}^{m} \sqrt{\pi} \frac{\Gamma(s) \Gamma(s-1 / 2)}{\Gamma(s+m / 2) \Gamma(s-m / 2)}
\end{aligned}
$$

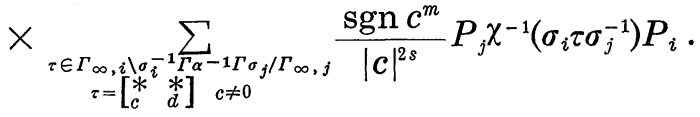

Now we calculate the contribution

$$
\frac{1}{8 \pi^{2}} \sum_{i=1}^{\infty} \int_{\Gamma \backslash \tilde{\boldsymbol{H}}^{*}} \int_{-\infty}^{\infty} h(r) F_{i}(z, \phi, s)^{t} \overline{E_{i}(z, \phi, s)} d r d z d \phi
$$


Using the Maass-Selberg type relation for this case, we get

$$
\begin{aligned}
& \frac{1}{2 \pi} \int_{\Gamma \backslash \overline{\boldsymbol{B}^{*}}} F_{i}(z, \phi ; s) \overline{E_{i}(z, \phi ; s)} d z d \phi \\
& \quad=\lim _{\sigma \rightarrow 1 / 2} \sum_{j=1}^{\infty}\left[\frac{w_{i j}(s)^{t} \overline{\delta_{i j} P_{i}} Y^{2 \sigma-1}-\varphi_{i j}^{*}(s)^{t} \overline{\varphi_{i j}(s)} Y^{1-2 \sigma}}{2 \sigma-1}\right] \\
& \quad+\lim _{\sigma \rightarrow 1 / 2} \sum_{j=1}^{\infty}\left[\frac{w_{i j}(s)^{\frac{t}{\varphi_{i j}(s)} Y^{2 \sqrt{-1} r}-\varphi_{i j}^{*}(s)^{\frac{t}{\delta_{i j}}} \overline{P_{i}} Y^{-2 \sqrt{-1} r}}}{2 \sqrt{-1} r}\right]+o(1),
\end{aligned}
$$

where $s=\sigma+\sqrt{-1} r$, and $o$-symbol is for $Y \rightarrow \infty$.

From the same formula, we can see

$$
\begin{aligned}
& w_{i i}\left(\frac{1}{2}+\sqrt{-1} x\right)=\sum_{j=1}^{\infty} \varphi_{i j}^{*}\left(\frac{1}{2}+\sqrt{-1} x\right)^{t} \varphi_{i j}\left(\frac{1}{2}+\sqrt{-1} x\right) \\
& \varphi_{i i}^{*}(x)=\sum_{j=1}^{\infty} w_{i j}(x)^{t} \overline{\varphi_{i j}(x)},
\end{aligned}
$$

for $x \in R$. The first part of (23) is

$$
\begin{aligned}
& \lim _{\sigma \rightarrow 1 / 2} \sum_{j=1}^{\infty}\left[\frac{w_{i j}(s)^{\bar{t} \delta_{i j} P_{i}} Y^{2 \sigma-1}-\varphi_{i j}^{*}(s)^{\bar{t}} \varphi_{i j}(s) Y^{1-2 \sigma}}{2 \sigma-1}\right] \\
& =\lim _{\sigma \rightarrow 1 / 2}\left[\frac{w_{i i}(s)\left(Y^{2 \sigma-1}-Y^{1-2 \sigma}\right)}{2 \sigma-1}-\frac{\left(w_{i \imath}(s)-\sum \varphi_{i j}^{*}(s)^{t} \overline{\varphi_{i j}(s)}\right) Y^{1-2 \sigma}}{2 \sigma-1}\right] \\
& =2 \log Y w_{i i}\left(\frac{1}{2}+\sqrt{-1} r\right) \\
& +\left.\frac{1}{2} \sum_{j=1}^{\infty} \frac{\partial}{\partial \sigma}\left(w_{i j}(s) \delta_{i j}-\varphi_{i j}^{*}(s)^{t} \overline{\varphi_{i j}(s)}\right)\right|_{\sigma=1 / 2} .
\end{aligned}
$$

Thus the contribution from the first part of (23) is

$$
\begin{aligned}
& \frac{1}{2 \pi} \log Y \int_{-\infty}^{\infty} h(r) w_{i i}\left(\frac{1}{2}+\sqrt{-1} r\right) d r \\
& \quad+\frac{1}{8 \pi} \int_{-\infty}^{\infty} h(r) \sum_{j=1}^{\infty}\left[\left.\frac{\partial}{\partial \sigma}\left(w_{i j}(s) \delta_{i j}-\varphi_{i j}^{*}(s)^{t} \varphi_{i j}(s)\right)\right|_{\sigma=1 / 2}\right] d r .
\end{aligned}
$$

Notice the fact

$$
\int_{-\infty}^{\infty} f(t) \frac{Y^{2 \sqrt{-1} t}}{\sqrt{-1} t} d t=\pi f(0) .
$$

This integral is considered as Cauchy's principal value. For the second part of (23), we have 


$$
\begin{aligned}
& \frac{1}{4 \pi} \int_{-\infty}^{\infty} h(r) \sum_{j=1}^{\infty}\left[\frac{w_{i j}(s) P_{i} t \overline{\varphi_{i j}(s)} Y^{2 \sqrt{-1} r}-\varphi_{i j}^{*}(s)^{t} \overline{\delta_{i j} P_{i}} Y^{-2 \sqrt{-1} r}}{2 \sqrt{-1} r}\right] d r \\
& =\frac{h(0)}{8} \sum_{j=1}^{\infty}\left[w_{i j}\left(\frac{1}{2}\right) \overline{{ }^{t}} \overline{\varphi_{i j}\left(\frac{1}{2}\right)}+\varphi_{i j}^{*}\left(\frac{1}{2}\right) t \overline{\delta_{i j} P_{i}}\right]+o(1) \\
& =\frac{h(0)}{4} \varphi_{i i}^{*}\left(\frac{1}{2}\right)+o(1)
\end{aligned}
$$

where $o$ is for $\sigma \rightarrow 1 / 2$. Combining these results to obtain

$$
\begin{aligned}
(17)= & \frac{\log Y}{2 \pi} \sum_{i=1}^{\infty} \sum_{\mu} g\left(2 \log \left|d_{\mu}\right|\right) \frac{\operatorname{sgn}\left(d_{\mu}\right)^{m}}{\left|d_{\mu}\right|} \operatorname{tr}\left\{\chi\left(\alpha_{\mu}\right) P_{i}\right\} \\
& +\frac{h(0)}{4} \sum_{i=1}^{\infty} \operatorname{tr} \varphi_{i i}^{*}\left(\frac{1}{2}\right) \\
& +\frac{1}{8 \pi} \int_{-\infty}^{\infty} h(r) \operatorname{tr} \sum_{i, j=1}^{\infty}\left[\left.\frac{\partial}{\partial \sigma}\left(w_{i j}(s) \delta_{i j}-\varphi_{i j}^{*}(s)^{t} \overline{\varphi_{i j}(s)}\right)\right|_{\sigma=1 / 2}\right] d r .
\end{aligned}
$$

Define $(\omega \times \nu) \times(\omega \times \nu)$ matrices $W(s), \Psi(s), \Psi^{*}(s)$ by

$$
W(s)=\left(w_{i j}(s)\right), \Psi(s)=\left(\varphi_{i j}(s)\right), \Psi^{*}(s)=\left(\varphi_{i j}^{*}(s)\right) .
$$

From the analogous relations to (24), we see

$$
\begin{gathered}
\left.\Psi(1 / 2+\sqrt{-1} r)^{t} \overline{\Psi(1 / 2+\sqrt{-1} r}\right)=\left(\delta_{i j} P_{i}\right) \\
\Psi^{*}(1 / 2+\sqrt{-1} r)^{t} \overline{\Psi(1 / 2+\sqrt{-1} r)}=W(1 / 2+\sqrt{-1} r) .
\end{gathered}
$$

By Liouville's theorem and (26), we obtain

$$
W(s) \Psi(s)=\Psi^{*}(s),
$$

for all $s \in C$. Let $h=\sigma-1 / 2$ and,

$$
\begin{aligned}
& W(s)=A+B h+o\left(h^{2}\right) \\
& \Psi(s)=C+D h+o\left(h^{2}\right) \\
& \Psi^{*}(s)=C_{1}+D_{1} h+o\left(h^{2}\right),
\end{aligned}
$$

as $h \rightarrow 0$. Then from (25), (26) and (27), we get

$$
A C=C_{1}, \quad A D+B C=D_{1}, \quad C^{t} \bar{C}=\left(\delta_{i j} P_{i}\right) .
$$

Using these facts, we have

$$
\begin{aligned}
\sum_{i, j=1}^{\infty} w_{i j}(s) \delta_{i j}-\varphi_{i j}^{*}(s)^{t} \overline{\varphi_{i j}(s)} \\
\quad=\operatorname{tr}\left(W(s)-\Psi^{* t} \bar{\Psi}\right) \\
\quad=-h \operatorname{tr}\left(A\left(D^{t} \bar{C}+C^{t} \bar{D}\right)\right)+o\left(h^{2}\right) .
\end{aligned}
$$


Finally we have

$$
\begin{aligned}
(19) & =\frac{\log Y}{2 \pi} \sum_{i=1}^{\infty} \sum_{\mu} g\left(2 \log \left|d_{\mu}\right|\right) \frac{\operatorname{sgn}\left(d_{\mu}\right)^{m}}{\left|d_{\mu}\right|} \operatorname{tr}\left\{\chi\left(\alpha_{\mu}\right) P_{i}\right\} \\
& +\frac{h(0)}{4} \sum_{i=1}^{\infty} \operatorname{tr} \varphi_{i i}^{*}\left(\frac{1}{2}\right) \\
& -\frac{1}{8 \pi} \int_{-\infty}^{\infty} h(r) \operatorname{tr}[W(1 / 2+\sqrt{-1} r) \\
& \times\left(\Psi^{\prime}(1 / 2+\sqrt{-1} r)^{t} \bar{\varphi}(1 / 2+\sqrt{-1} r)+\Psi(1 / 2+\sqrt{-1} r)^{t} \overline{\left.\Psi^{\prime}(1 / 2+\sqrt{-1} r)\right)}\right] d r .
\end{aligned}
$$

Considering the conjugacy classes of $\Gamma \alpha \Gamma$ with respect to $\Gamma$ which fix cusps of $\Gamma$ precisely, we see that all $\log Y$ terms cancel out in (15). Especially in the case (IV), there is a certain difference between the element which fix equivalent cusps and the one which fix inequivalent cusps.

Theorem 3. The contribution from $H\left(z, \phi, z^{\prime}, \phi^{\prime}\right)$ is given by

$$
\begin{aligned}
& \frac{h(0)}{4} \operatorname{tr} W\left(\frac{1}{2}\right) \Phi\left(\frac{1}{2}\right) \\
& \quad-\frac{1}{4 \pi} \int_{-\infty}^{\infty} h(r) \operatorname{tr}\left[W(1 / 2+\sqrt{-1} r) \Psi^{\prime}(1 / 2+\sqrt{-1} r) \Psi(1 / 2-\sqrt{-1} r)\right] d r .
\end{aligned}
$$

Notice that this contribution appears with the opposite sign in (15).

Remark. The above results are also valid when $\Gamma$ contains -1 . Because each step of the computation is almost the same, we don't repeat it. But in the next section we consider, as an example, the case $\Gamma=\Gamma_{0}(p)$ and write down the terms of $\S 4-\S 6$ as explicitly as possible.

\section{§. The case $\Gamma=\Gamma_{0}(p)$}

We assume that $\Gamma=\Gamma_{0}(p), p$ a prime, and consider a double coset $B=\Gamma\left(\frac{1}{\sqrt{D}}\left(\begin{array}{ll}1 & 0 \\ 0 & D\end{array}\right)\right) \Gamma$ for $D \in Z, D>0$ and $(D, p)=1$. Let $\chi$ be a Dirichlet character $\bmod p$ such that $\chi(-1)=(-1)^{m}$. We also assume for simplicity that $\chi$ is non-trivial. We put $\chi(g)=\chi(d)$ for $g=\frac{1}{\sqrt{D}}\left(\begin{array}{ll}a & b \\ c & d\end{array}\right) \in B$. We take

$$
\sigma_{\infty}=1 \quad \text { and } \quad \sigma_{0}=\frac{1}{\sqrt{p}}\left(\begin{array}{rr}
0 & -1 \\
p & 0
\end{array}\right) .
$$


(i) Parabolic conjugacy classes

Parabolic conjugacy classes exist only when $D$ is a square. We put $a=\sqrt{D} \in Z$. Then the complete representatives of parabolic conjugacy classes are

$$
P_{\infty} \cup P_{0}
$$

where $P_{\infty}=\{g \in B \mid g$ is parabolic and $g \infty=\infty\}=\left\{ \pm\left(\begin{array}{cc}1 & b / a \\ 0 & 1\end{array}\right) \mid b \in Z,(a, b)=1\right\}$ and $P_{0}=\sigma_{0} P_{\infty} \sigma_{0}^{-1}$. Hence in the notation of $\S 4$, we have $\mu=b / a, \zeta=0$ and $r_{i}=1$. Noting that $\Gamma$ contains -1 , we get the parabolic contribution as

where

$$
2 \chi(a)^{-1} \sum_{\substack{0<b<a \\(a, b)=1}} g(0) \log Y+J(P)+o(1)
$$

$$
\begin{gathered}
J(P)=2 \chi(a)^{-1} \sum_{\substack{0<b<a \\
(a, b)=1}}\left\{-g(0)\left(\frac{\Gamma^{\prime}}{\Gamma}(b / a)+\gamma+\log 2\right)+\frac{1}{4} h(0)\right. \\
\left.-\frac{1}{2 \pi} \int_{-\infty}^{\infty} h(r) \frac{\Gamma^{\prime}}{\Gamma}(1+\sqrt{-1} r) d r+\int_{0}^{\infty} \frac{g(u)}{2 \sinh (u / 2)}\left(1-\cosh \left(\frac{m u}{2}\right)\right) d u\right\} .
\end{gathered}
$$

(ii) Hyperbolic conjugacy classes which fix cusps of $\Gamma$.

Let $H$ be the set of all hyperbolic elements in $B$ which fix cusps of $\Gamma$. Let $\mathrm{g}$ be an element of $H$ and $\eta_{1}, \eta_{2}$ be the fixed points of $g$. By the assumption, $\eta_{1}$ and $\eta_{2}$ are cusps of $\Gamma$. The following three cases occur:

$$
\eta_{1} \sim \eta_{2} \sim \infty, \eta_{1} \sim \eta_{2} \sim 0, \text { and } \eta_{1} \sim \infty, \eta_{2} \sim 0 \text {. }
$$

For $\kappa=\infty$ or 0 , we put $H_{k}=\{g \in H \mid g \kappa=\kappa\}$. First we consider $H_{\infty}$. We can easily see that the representatives of conjugacy classes $H_{\infty} / \sim$ are given by $\mathscr{R}_{1} \cup \mathscr{R}_{2}$ where

$\mathscr{R}_{1}=\left\{ \pm \frac{1}{\sqrt{D}}\left(\begin{array}{ll}a & b \\ 0 & d\end{array}\right) \mid \begin{array}{l}a d=D, a \neq d, a>0,(a, b, d)=1, b \bmod |a-d| \\ (a-d) /(b, a-d) \neq \equiv 0 \bmod p\end{array}\right\}$

and

$\mathscr{R}_{2}=\left\{ \pm \frac{1}{\sqrt{D}}\left(\begin{array}{ll}a & b \\ 0 & d\end{array}\right) \mid \begin{array}{l}a d=D, a \neq d, a>0,(a, b, d)=1, b \bmod |a-d| \\ (a-d) /(b, a-d) \equiv 0 \bmod p\end{array}\right\} / \sim$.

One remark is necessary for $\mathscr{R}_{2}$. For each $g=\frac{1}{\sqrt{D}}\left(\begin{array}{ll}a & b \\ 0 & d\end{array}\right)$ with the above condition, there exists a unique $g=\frac{1}{\sqrt{D}}\left(\begin{array}{ll}d & b^{\prime} \\ 0 & a\end{array}\right)$ with the same condition 
such that $g \sim g^{\prime}$. Therefore we identify $g$ and $g^{\prime}$.

Let $T=\frac{1}{\sqrt{D}}\left(\begin{array}{ll}a & b \\ 0 & d\end{array}\right)$ be an element of $\mathscr{R}_{1}\left(\right.$ resp. $\left.\mathscr{R}_{2}\right)$. Then the fixed points of $T$ are $\eta_{1}=\infty$ and $\eta_{2}=b /(d-a) \sim 0$ (resp. $\left.\infty\right)$. In the notation of $\S 4$, we have

$$
\varphi=\left(\begin{array}{cc}
1 & -\eta_{2} \\
0 & 1
\end{array}\right) \text { and } c(\tau)= \pm \sqrt{p} \frac{a-d}{(b, a-d)}\left(\text { resp. } \pm \frac{a-d}{(b, a-d)}\right) .
$$

Since $\sigma_{0} \Gamma \sigma_{0}^{-1}=\Gamma$, the contribution from the classes in $H_{0}$ is the same from the classes in $H_{\infty}$. Summing up, the contribution from $H / \sim$ is given by

$$
2 \sqrt{D} \sum_{\substack{a d d=D \\ a>d>0}}\left(\chi(a)^{-1}+\chi(d)^{-1}\right) \frac{\varphi((a, d))}{(a, d)} g\left(w_{0}\right) \log Y+J(H)+o(1)
$$

where

$$
\begin{aligned}
J(H)= & \sqrt{D} \sum_{\substack{a d=D \\
a>d>0}}\left(\chi(a)^{-1}+\chi(d)^{-1}\right)\left\{\frac{\varphi((a, d))}{(a, d)} G_{a, d}(m)\right. \\
& \left.+\frac{1}{a-d} g\left(w_{0}\right) \sum_{\substack{b<b<a-d \\
0, b, b)=1}} \log \left(\sqrt{p} \frac{a-d}{(a-d, b)}\right)^{2}\right\},
\end{aligned}
$$

with

$$
\begin{aligned}
G_{a, d}(m)= & \int_{w_{0}}^{\infty} g(u)\left[\frac{\cosh (u / 2)}{\sinh (u / 2)+\sinh \left(w_{0} / 2\right)}\right. \\
& \left.+\frac{\left(1-\cosh \left(\left(u-w_{0}\right) m / 2\right) \sinh \left(\left(u+w_{0}\right) / 2\right)\right.}{(\sinh (u / 2))^{2}-\left(\sinh \left(w_{0} / 2\right)\right)^{2}}\right] d u
\end{aligned}
$$

and

$$
w_{0}=\log (a / d)
$$

(iii) Eisenstein part

The constant term of the Fourier expansion of Eisenstein series is determined in [6] Chapter 11, §4 and [12], i.e.

$$
\begin{gathered}
\varphi_{\infty, \infty}=\varphi_{0,0}=0, \\
\varphi_{\infty, 0}=\frac{(-\sqrt{-1})^{m} \sqrt{\pi} \Gamma(s) \Gamma(s-1 / 2)}{p^{s} \Gamma(s+m / 2) \Gamma(s-m / 2)} \frac{L(2 s-1, \bar{\chi})}{L(2 s, \bar{\chi})}, \\
\varphi_{0, \infty}=\frac{\sqrt{-1}^{m} \sqrt{\pi} \Gamma(s) \Gamma(s-1 / 2)}{p^{s} \Gamma(s+m / 2) \Gamma(s-m / 2)} \frac{L(2 s-1, \chi)}{L(2 s, \chi)} .
\end{gathered}
$$


We can see easily that $w_{\infty, 0}=w_{0, \infty}=0$ and

$$
\begin{aligned}
& w_{\infty, \infty}(s)=D^{s} \sum_{\substack{a d=D \\
d>0}} \frac{\varphi((a, d))}{(a, d)} \frac{\chi(d)^{-1}}{d^{2 s-1}}, \\
& w_{0,0}(s)=D^{s} \sum_{\substack{a d=D \\
d>0}} \frac{\varphi((a, d))}{(a, d)} \frac{\chi(a)^{-1}}{d^{2 s-1}} .
\end{aligned}
$$

We have, by (31), (32) and the functional equation of $L$ and digamma functions, that

$$
\begin{aligned}
\frac{\partial}{\partial \sigma} \log \varphi_{\infty}(1 / 2+\sqrt{-1} r) \\
=\log \left(\pi^{2} / p^{3}\right)-\frac{\Gamma^{\prime}}{\Gamma}\left(\frac{1+m}{2}+\sqrt{-1} r\right)-\frac{\Gamma^{\prime}}{\Gamma}\left(\frac{1+m}{2}-\sqrt{-1} r\right) \\
\quad-2\left\{\frac{L^{\prime}}{L}(1-2 \sqrt{-1} r, \chi)+\frac{L^{\prime}}{L}(1+2 \sqrt{-1} r, \bar{\chi})\right\}
\end{aligned}
$$

and

$$
\begin{aligned}
& \frac{\partial}{\partial \sigma} \log \varphi_{0 \infty}(1 / 2+\sqrt{-1} r) \\
& =\log \left(\pi^{2} / p^{3}\right)-\frac{\Gamma^{\prime}}{\Gamma}\left(\frac{1+m}{2}+\sqrt{-1} r\right)-\frac{\Gamma^{\prime}}{\Gamma}\left(\frac{1+m}{2}-\sqrt{-1} r\right) \\
& \quad-2\left\{\frac{L^{\prime}}{L}(1-2 \sqrt{-1} r, \bar{\chi})+\frac{L^{\prime}}{L}(1+2 \sqrt{-1} r, \chi)\right\} .
\end{aligned}
$$

Using these facts, we get a contribution from Eisenstein series as

$$
\left.\sqrt{D} \log Y \sum_{\substack{a d=D \\ d>0}} \frac{\varphi((a, d))}{(a, d)}\left(\chi(a)^{-1}+\chi(d)\right)^{-1}\right) g(\log (a / d))+J(C)+o(1),
$$

where

$$
\begin{aligned}
J(C)= & -\frac{1}{4 \pi} \int_{-\infty}^{\infty} h(r)\left[w_{\infty \infty}(1 / 2+\sqrt{-1} r) \operatorname{Re} \frac{\partial}{\partial \sigma} \log \varphi_{\infty 0}(1 / 2+\sqrt{-1} r)\right. \\
& \left.+w_{00}(1 / 2+\sqrt{-1} r) \operatorname{Re} \frac{\partial}{\partial \sigma} \log \varphi_{0 \infty}(1 / 2+\sqrt{-1} r)\right] d r \\
= & -\frac{\sqrt{D}}{2 \pi} \int_{-\infty}^{\infty} h(r)\left[\sum_{\substack{a d=D \\
a>0}} \frac{\varphi((a, d))}{(a, d)} \chi(d)^{-1}(a / d)^{\sqrt{-1} r}\right]\left[\log \left(\pi^{2} / p^{3}\right)\right. \\
- & \frac{\Gamma^{\prime}}{\Gamma}\left(\frac{1+m}{2}+\sqrt{-1} r\right)-\frac{\Gamma^{\prime}}{\Gamma}\left(\frac{1+m}{2}-\sqrt{-1} r\right) \\
- & \left.2 \frac{L^{\prime}}{L}(1-2 \sqrt{-1} r, \chi)-2 \frac{L^{\prime}}{L}(1+2 \sqrt{-1} r, \bar{\chi})\right] d r .
\end{aligned}
$$


To state the theorem, put

$$
\begin{aligned}
& J(E)=\sum_{[R]: \text { elliptic }} I(R) \\
& J(h)=\sum_{[T]} I(T)
\end{aligned}
$$

where the last summation is over hyperbolic conjugacy classes which fix hyperbolic points of $\Gamma$.

Theorem 4. Under the notations of (18), (28), (29), (33), (34), and (35), the following Selberg trace formula holds for $B=\Gamma \frac{1}{\sqrt{D}}\left(\begin{array}{ll}1 & 0 \\ 0 & D\end{array}\right) \Gamma$ :

$$
\begin{array}{rl}
\sum_{j} & h\left(\sqrt{-1 / 4-\lambda_{j}}\right) \operatorname{tr}\left(T(B) ; \lambda_{j}\right) \\
& =J(\mathrm{id})+J(-\mathrm{id})+J(E)+J(h)+J(H)+J(P)-J(C) .
\end{array}
$$

In the rest of this paper, we will treat the functional equation of the Selberg type zeta function for a double coset. Although we can derive it for general Fuchsian group $\Gamma$, we restrict ourselves to the group $\Gamma_{0}(p)$ since we can describe the Eisenstein part by the Dirichlet $L$-function.

For $G_{a, a}(m)$, we have

Lemma 7. Let $m$ be an integer. Suppose that there is a constant $A \gg 0$ such that $g(u)=O\left(e^{-A u}\right)$ for $u \rightarrow \infty$. Then we have

$$
\begin{aligned}
G_{a, d}(m)-G_{a, d}(m-2)= & \frac{m-1}{2 \pi} \int_{-\infty}^{\infty} h(r)\left(\frac{a}{d}\right)^{-\sqrt{-1} r} \frac{1}{r^{2}+((m-1) / 2)^{2}} d r \\
& -\operatorname{sgn}(m-1) h\left(\frac{m-1}{2} \sqrt{-1}\right)\left(\frac{a}{d}\right)^{\operatorname{sgn}(m-1) \cdot(1-m) / 2}
\end{aligned}
$$

Proof. By (30), we have

$$
\begin{aligned}
G_{a, d}(m)-G_{a, d}(m-2) & =\int_{w_{0}}^{\infty} g(u)\left(e^{(1 / 2)\left(u-w_{0}\right)(1-m)}-e^{(1 / 2)\left(u-w_{0}\right)(m-1)}\right) d u \\
& =\int_{0}^{\infty} g\left(u+w_{0}\right) e^{u(1-m) / 2} d u-\int_{-\infty}^{\infty} g\left(u+w_{0}\right) e^{u(m-1) / 2} d u .
\end{aligned}
$$

We denote the former integral by $\tilde{\boldsymbol{g}}_{1}(m)$ and the latter one by $\tilde{\boldsymbol{g}}_{2}(m)$. To express these integrals by $h(r)$, we consider $m$ as a complex variable. Firstly we assume that $\operatorname{Re}(m)>1$. Substituting (14) and interchanging the integrals, we have

$$
\tilde{g}_{1}(m)=-\frac{1}{2 \pi} \int_{-\infty}^{\infty} h(r) \frac{e^{-\sqrt{-1} r w_{0}}}{(1-m) / 2-\sqrt{-1} r} d r .
$$


The function $\tilde{g}_{1}(m)$, defined by (37) for $\operatorname{Re}(m)>1$, can be continued meromorphically over the line $\operatorname{Re}(m)=1$ and for $\operatorname{Re}(m)<1$,

$$
\tilde{g}_{1}(m)=-\frac{1}{2 \pi} \int_{-\infty}^{\infty} h(r) \frac{e^{-\sqrt{-1} r w_{0}}}{(1-m) / 2-\sqrt{-1} r} d r+h\left(\frac{m-1}{2} \sqrt{-1}\right) e^{w_{0}(m-1) / 2} .
$$

Similarly we have

$$
\tilde{g}_{2}(m)=-\frac{1}{2 \pi} \int_{-\infty}^{\infty} h(r) \frac{e^{-\sqrt{-1} r w_{0}}}{(m-1) / 2-\sqrt{-1} r} d r
$$

for $\operatorname{Re}(m)<1$. When (38) is continued to the region such that $\operatorname{Re}(m)>1$, we have

$$
\tilde{g}_{2}(m)=-\frac{1}{2 \pi} \int_{-\infty}^{\infty} h(r) \frac{e^{-\sqrt{-1} r w_{0}}}{(m-1) / 2-\sqrt{-1} r} d r+h\left(\frac{1-m}{2} \sqrt{-1}\right) e^{w_{0}(1-m) / 2} .
$$

Hence we get the lemma.

Now we define the Selberg type zeta function.

Definition. The Selberg type zeta function $z_{B}(\alpha)$ with respect to a double coset $B$ is defined by

$$
z_{B}(\alpha)=\sum_{[T]} \frac{(\operatorname{sgn} T)^{m} \chi(T) \log \left(\delta_{0}\right)}{\delta^{1 / 2}-\delta^{-1 / 2}} \delta^{-\alpha} .
$$

Here the summation runs over the hyperbolic conjugacy classes of $B /\{ \pm 1\}$ which fix hyperbolic points of $\Gamma$. If $T$ and $T_{0}$ are as in $\S 2$, then $\delta=\lambda^{2}$ and $\delta_{0}=\lambda_{0}^{2}$.

Theorem 5. Let $B=\Gamma\left(\frac{1}{\sqrt{D}}\left(\begin{array}{ll}1 & 0 \\ 0 & D\end{array}\right)\right) \Gamma, D$ a positive integer which is prime to $p$. Then $z_{B}(\alpha)$ satisfies the following functional equation

$$
\begin{aligned}
& -\frac{\chi(D)^{-1}}{2}\left({ }_{\partial_{B}}(\alpha)+z_{B}(-\alpha)\right) \\
= & -\delta(D) \frac{(p+1) \pi \alpha}{6} \frac{\sin (2 \pi \alpha)}{\cos (2 \pi \alpha)+(-1)^{m}} \\
& +\frac{\pi}{2} \sum_{[R]}\left\{\begin{array}{l}
\frac{\chi(R)}{\# \Gamma(R) \sin \theta} \frac{\cos (\pi-2 \theta) \alpha}{\cos \pi \alpha} \quad \text { (if } m \text { is even) } \\
\left.\frac{\chi(R)}{\sharp \Gamma(R) \sin \theta} \frac{\sin (\pi-2 \theta) \alpha}{\sin \pi \alpha} \quad \text { (if } m \text { is odd }\right)
\end{array}\right. \\
& -2 \chi(\sqrt{D})^{-1} \sum_{\substack{0<b<\sqrt{D} \bar{D} \\
(b, \sqrt{D})=1}} \frac{\Gamma^{\prime}}{\Gamma}(b / \sqrt{D})+2 \chi(\sqrt{D})^{-1} \varphi(\sqrt{D})(\log 2-\gamma)
\end{aligned}
$$




$$
\begin{aligned}
& +\chi(\sqrt{D})^{-1} \varphi(\sqrt{D})\left\{\log \frac{\pi^{2}}{p^{3}}-2 \frac{\Gamma^{\prime}}{\Gamma}(2 \alpha)-2 \frac{\Gamma^{\prime}}{\Gamma}(-2 \alpha)\right. \\
& \left.-\frac{L^{\prime}}{L}(1-2 \alpha, \chi)-\frac{L^{\prime}}{L}(1+2 \alpha, \chi)-\frac{L^{\prime}}{L}(1-2 \alpha, \bar{\chi})-\frac{L^{\prime}}{L}(1+2 \alpha, \bar{\chi})\right\} \\
& +\sqrt{D} \sum_{\substack{a d=D \\
a>d}}\left(\chi(a)^{-1}+\chi(d)^{-1}\right)\left[\frac { \varphi ( ( a , d ) ) } { ( a , d ) } \left\{\frac{1}{2}\left(\tilde{G}_{a, d, \alpha}\left(\varepsilon_{m}\right)-\tilde{G}_{a, d,-\alpha}\left(\varepsilon_{m}\right)\right)\right.\right. \\
& \left.+\cosh \left(\alpha w_{0}\right)\left(\log \frac{\pi^{2}}{p^{3}}-\frac{\Gamma^{\prime}}{\Gamma}\left(\frac{\varepsilon_{m}+1}{2}+\alpha\right)-\frac{\Gamma^{\prime}}{\Gamma}\left(\frac{\varepsilon_{m}+1}{2}-\alpha\right)\right)\right\} \\
& \left.+\frac{\cosh \left(\alpha w_{0}\right)}{(a-d)} \sum_{b} 2 \log \left(\sqrt{p} \frac{a-d}{(a-d, b)}\right)\right] \\
& -\sqrt{D} \sum_{\substack{a d=D \\
a>d}} \frac{\varphi((a, d))}{(a, d)}\left\{( \chi ( d ) ^ { - 1 } ( \frac { a } { d } ) ^ { \alpha } + \chi ( a ) ^ { - 1 } ( \frac { d } { a } ) ^ { \alpha } ) \left(\frac{L^{\prime}}{L}(1-2 \alpha, \chi)+\right.\right. \\
& \left.+\frac{L^{\prime}}{L}((1+2 \alpha, \bar{\chi}))+\left(\chi(d)^{-1}\left(\frac{a}{d}\right)^{-\alpha}+\chi(a)^{-1}\left(\frac{d}{a}\right)^{-\alpha}\right)\left(\frac{L^{\prime}}{L}(1+2 \alpha, \chi)+\frac{L^{\prime}}{L}(1-2 \alpha, \bar{\chi})\right)\right\}
\end{aligned}
$$

where

$$
\begin{aligned}
\delta(D) & = \begin{cases}1 & \text { if } D=1 \\
0 & \text { if } D>1,\end{cases} \\
\varepsilon_{m} & = \begin{cases}1 & \text { if } m \text { is odd } \\
0 & \text { if } m \text { is even },\end{cases} \\
w_{0} & =\log (a / d) \text { for each } a, b
\end{aligned}
$$

and, for $\operatorname{Re}(\alpha)>0, \tilde{G}_{a, a, \alpha}\left(\varepsilon_{m}\right)$ are defined by

$$
\begin{aligned}
& \tilde{G}_{a, a, \alpha}(0)=\int_{w_{0}}^{\infty} \frac{e^{-\alpha u} \cosh (u / 2)}{\sinh (u / 2)+\sinh \left(w_{0} / 2\right)} d u, \\
& \tilde{G}_{a, a, \alpha}(1)=\cosh \left(w_{0} / 2\right) \int_{w_{0}}^{\infty} \frac{e^{-\alpha u}}{\sinh (u / 2)+\sinh \left(w_{0} / 2\right)} d u .
\end{aligned}
$$

Also $\chi(\sqrt{D})$ and $\varphi(\sqrt{D})$ should be considered to be zero if $\sqrt{\bar{D}}$ is not an integer.

Proof. We select the Selberg kernel for $h(r)$ in the trace formula. Namely we put

$$
h_{\alpha}(r)=\frac{1}{r^{2}+\alpha^{2}}-\frac{1}{r^{2}+\beta^{2}}, \quad \operatorname{Re}(\alpha), \operatorname{Re}(\beta) \gg 0 .
$$

Its Fourier transform is given by

$$
g_{\alpha}(u)=\frac{1}{2 \alpha} e^{-\alpha|u|}-\frac{1}{2 \beta} e^{-\beta|u|} .
$$


Hence the hyperbolic term which fix hyperbolic points of $\Gamma$ reduces to $(1 / 2 \alpha) \chi(D)^{-1} z_{B}(\alpha)$. Each term of the right hand side of (36) is easily seen to be continued analytically to the whole complex plane (with respect to the variable $\alpha$ ). Since the left hand side of (36) is uniformly convergent on any compact set not containing any $\pm \sqrt{1 / 4+\lambda_{j}}$, it is unchanged if we substitute $-\alpha$ for $\alpha$.

Therefore we have

$$
\begin{aligned}
- & \frac{1}{2 \alpha} \chi(D)^{-1}\left(z_{B}(\alpha)+z_{B}(-\alpha)\right) \\
= & J_{\alpha}( \pm \mathrm{id})-J_{-\alpha}( \pm \mathrm{id})-J_{\alpha}(E)-J_{-\alpha}(E)+J_{\alpha}(H)-J_{-\alpha}(H) \\
& +J_{\alpha}(P)-J_{-\alpha}(P)-J_{\alpha}(C)+J_{-\alpha}(C)
\end{aligned}
$$

We put

$$
f_{m}(\alpha)= \begin{cases}\frac{1}{\alpha^{2}-(1 / 2)^{2}}+\frac{3}{\alpha^{2}-(3 / 2)^{2}}+\cdots+\frac{m-1}{\alpha^{2}-((m-1) / 2)^{2}} & \text { if } m \text { is even } \\ \frac{2}{\alpha^{2}-1^{2}}+\frac{4}{\alpha^{2}-2^{2}}+\cdots+\frac{m-1}{\alpha^{2}-((m-1) / 2)^{2}} & \text { if } m \text { is odd } .\end{cases}
$$

Identity, elliptic and parabolic terms can be calculated as follows:

$$
\begin{aligned}
& J_{\alpha}( \pm \mathrm{id})-J_{-\alpha}( \pm \mathrm{id})=-\delta(D) \frac{(p+1) \pi}{6} \frac{\sin (2 \pi \alpha)}{\cos (2 \pi \alpha)+(-1)^{m}}, \\
& J_{\alpha}(E)-J_{-\alpha}(E)= \frac{\pi}{2 \alpha} \sum_{\{R\}}\left\{\begin{array}{l}
\frac{\chi(R)}{\# \Gamma(R) \sin \theta} \frac{\cos (\pi-2 \theta) \alpha}{\cos \pi \alpha} \quad \text { if } m \text { is even } \\
\frac{\chi(R)}{\# \Gamma(R) \sin \theta} \frac{\sin (\pi-2 \theta) \alpha}{\sin \pi \alpha} \quad \text { if } m \text { is odd }
\end{array}\right. \\
& J_{\alpha}(P)-J_{-\alpha}(P)=2 \chi(\sqrt{D})^{-1} \sum_{\substack{0<b<\sqrt{D} \\
(b, \sqrt{D})=1}}\left\{-\frac{1}{\alpha}\left(\frac{\Gamma^{\prime}}{\Gamma}(b / \sqrt{D})+\gamma+\log 2\right)-\frac{1}{2 \alpha} f_{m}(\alpha)\right. \\
&\left.-\frac{1}{2 \alpha}\left(\frac{\Gamma^{\prime}}{\Gamma}\left(\frac{\varepsilon_{m}}{2}+\alpha\right)+\frac{\Gamma^{\prime}}{\Gamma}\left(\frac{\varepsilon_{m}}{2}-\alpha\right)\right)\right\},
\end{aligned}
$$

where $\varepsilon_{m}$ is defined as above (c.f. Hejhal [6] pp. 430-444). We consider $J_{\alpha}(H)$. We ignore the $\beta$-term in $h(r)$ or $g(u)$ for a while. By Lemma 7, we have

$$
\begin{aligned}
G_{a, a}(m) & =G_{a, d}(m-2)-\frac{e^{-\alpha w_{0}}}{2 \alpha} \frac{m-1}{\alpha^{2}-((m-1) / 2)^{2}} \\
& =\frac{1}{2 \alpha} \tilde{G}_{a, d, \alpha}\left(\varepsilon_{m}\right)-\frac{e^{-\alpha w_{0}}}{2 \alpha} f_{m}(\alpha)
\end{aligned}
$$


Clearly $\tilde{G}_{a, a, \alpha}\left(\varepsilon_{m}\right)$ can be continued to the whole $\alpha$-plane as a meromorphic function. Hence we have

$$
\begin{aligned}
J_{\alpha}(H)-J_{-\alpha}(H)= & \sqrt{D} \sum_{\substack{a d=D \\
a>d>0}}\left(\chi(a)^{-1}+\chi(d)^{-1}\right)\left[\frac { \varphi ( ( a , d ) ) } { ( a , d ) } \left\{\frac { 1 } { 2 \alpha } \left(\tilde{G}_{a, d, \alpha}\left(\varepsilon_{m}\right)\right.\right.\right. \\
& \left.\left.-\tilde{G}_{a, d,-\alpha}\left(\varepsilon_{m}\right)\right)-\frac{\cosh \left(\alpha w_{0}\right)}{\alpha} f_{m}(\alpha)\right\} \\
& \left.+\frac{\cosh \left(\alpha w_{0}\right)}{(a-d) \alpha} \sum_{b} 2 \log \left(\sqrt{p} \frac{a-d}{(a-d, b)}\right)\right] .
\end{aligned}
$$

Next we consider $J_{\alpha}(C)$. We also ignore the $\beta$-term in the following formula. By the residue theorem, we have, for $\operatorname{Re}(\alpha)>0$,

$$
\begin{aligned}
J_{\alpha}(C)= & -\frac{\varphi(\sqrt{D})}{2 \pi} \chi(\sqrt{D})^{-1} \frac{\pi}{\alpha}\left\{\log \frac{\pi^{2}}{p^{3}}-\left(\frac{\Gamma^{\prime}}{\Gamma}\left(\frac{m+1}{2}+\alpha\right)+\frac{\Gamma^{\prime}}{\Gamma}\left(\frac{m+1}{2}-\alpha\right)\right)\right\} \\
& +\frac{\varphi(\sqrt{D})}{\pi} \chi(\sqrt{D})^{-1} \int_{-\infty}^{\infty} h(r)\left(\frac{L^{\prime}}{L}(1-2 \sqrt{-1} r, \chi)+\frac{L^{\prime}}{L}(1+2 \sqrt{-1} r, \bar{\chi})\right) d r \\
& -\frac{\sqrt{D}}{2 \pi} \sum_{\substack{a d=D \\
a>d}} \frac{\varphi((a, d))}{(a, d)}\left(\chi(a)^{-1}+\chi(d)^{-1}\right)(a / d)^{-\alpha} \frac{\pi}{\alpha}\left\{\log \frac{\pi^{2}}{p^{3}}-\right. \\
& \left.-\left(\frac{\Gamma^{\prime}}{\Gamma}\left(\frac{m+1}{2}+\alpha\right)+\frac{\Gamma^{\prime}}{\Gamma}\left(\frac{m+1}{2}-\alpha\right)\right)\right\} \\
& +\frac{\sqrt{D}}{\pi} \sum_{\substack{a d=D \\
a>d}} \frac{\varphi((a, d))}{(a, d)}\left\{\int_{-\infty}^{\infty} h(r)\left(\chi(d)^{-1}(a / d)^{\sqrt{-1} r}+\chi(a)^{-1}(d / a)^{\sqrt{-1} r}\right)\right. \\
& \left.\times\left(\frac{L^{\prime}}{L}(1-2 \sqrt{-1} r, \chi)+\frac{L^{\prime}}{L}(1+2 \sqrt{-1} r, \bar{\chi})\right) d r\right\} .
\end{aligned}
$$

These integrals can be continued meromorphically to the whole $\alpha$-plane with a simple pole at $\alpha=0$.

Therefore

$$
\begin{gathered}
J_{\alpha}(C)-J_{-\alpha}(C)=-\frac{\varphi(\sqrt{D}) \chi(\sqrt{D})^{-1}}{\alpha}\left\{\log \frac{\pi^{2}}{p^{3}}-\left(\frac{\Gamma^{\prime}}{\Gamma}\left(\frac{m+1}{2}+\alpha\right)+\frac{\Gamma^{\prime}}{\Gamma}\left(\frac{m+1}{2}-\alpha\right)\right)\right\} \\
-\frac{\sqrt{D}}{\alpha} \sum_{\substack{a d=D \\
a>d}} \frac{\varphi((a, d))}{(a, d)}\left(\chi(\alpha)^{-1}+\chi(d)^{-1}\right) \cosh \left(\alpha w_{0}\right) \\
\times\left\{\log \frac{\pi^{2}}{p^{3}}-\left(\frac{\Gamma^{\prime}}{\Gamma}\left(\frac{m+1}{2}+\alpha\right)+\frac{\Gamma^{\prime}}{\Gamma}\left(\frac{m+1}{2}-\alpha\right)\right)\right\} \\
+\frac{\varphi(\sqrt{D})}{\alpha} \chi(\sqrt{D})^{-1}\left(\frac{L^{\prime}}{L}(1-2 \alpha, \chi)+\frac{L^{\prime}}{L}(1+2 \alpha, \chi)+\frac{L^{\prime}}{L}(1-2 \alpha, \bar{\chi})+\frac{L^{\prime}}{L}(1+2 \alpha, \bar{\chi})\right) \\
+\frac{\sqrt{D}}{\alpha} \sum_{\substack{a d=D \\
a>d}} \frac{\varphi((a, d))}{(a, d)}\left\{( \chi ( d ) ^ { - 1 } ( \frac { a } { d } ) ^ { \alpha } + \chi ( a ) ^ { - 1 } ( \frac { d } { a } ) ^ { \alpha } ) \left(\frac{L^{\prime}}{L}(1-2 \alpha, \chi)\right.\right.
\end{gathered}
$$




$$
\begin{array}{r}
\left.+\frac{L^{\prime}}{L}(1+2 \alpha, \bar{\chi})\right)+\left(\chi(d)^{-1}\left(\frac{a}{d}\right)^{-\alpha}+\chi(a)^{-1}\left(\frac{d}{a}\right)^{-\alpha}\right) \\
\left.\times\left(\frac{L^{\prime}}{L}(1+2 \alpha, \chi)+\frac{L^{\prime}}{L}(1-2 \alpha, \bar{\chi})\right)\right\} .
\end{array}
$$

Because

$$
\frac{\Gamma^{\prime}}{\Gamma}\left(\frac{m+1}{2}+\alpha\right)+\frac{\Gamma^{\prime}}{\Gamma}\left(\frac{m+1}{2}-\alpha\right)=\frac{\Gamma^{\prime}}{\Gamma}\left(\frac{\varepsilon_{m}+1}{2}+\alpha\right)+\frac{\Gamma^{\prime}}{2}\left(\frac{\varepsilon_{m}+1}{2}-\alpha\right)-f_{m}(\alpha),
$$

we get the Theorem.

Remark. Let $m=1$ and consider the space $L_{x}(1,-1 / 4)$. It is isomorphic to the space $S_{1}(\Gamma, \chi)$ by Lemma 1 . We also empoly the Selberg kernel for $h(r)$. After multiplying the trace formula for $B=\Gamma \frac{1}{\sqrt{D}}\left(\begin{array}{ll}1 & 0 \\ 0 & D\end{array}\right) \Gamma$ by $\alpha$, take the residue at $\alpha=0$. Then we get

$$
\operatorname{tr} T(B,-1 / 4)=\frac{\chi(D)^{-1}}{2} \operatorname{Res}_{\alpha=0} z_{B}(\alpha)+\chi(D)^{-1} \frac{\sqrt{-1}}{4} \sum_{\{R\}} \frac{\chi(R)}{\sharp \Gamma(R) \sin \theta} .
$$

The last sum is equal to zero, because $R$ and $\left(\begin{array}{rr}-1 & 0 \\ 0 & 1\end{array}\right) R\left(\begin{array}{rr}-1 & 0 \\ 0 & 1\end{array}\right)$ belong to the different conjugacy classes. Hence tr $T(B,-1 / 4)=\left(\chi(D)^{-1} / 2\right) \operatorname{Res}_{\alpha=0} z_{B}(\alpha)$.

\section{REFERENCES}

[1] S. Akiyama, Selberg trace formula for odd weight I, II, Proc. Japan Acad., 64A, no. $9,10,341-344,387-388$.

[2] S. Akiyama, Y. Tanigawa, T. Hiramatsu, On traces of Hecke operators for the case of weight one (in Japanese), Automorphic Forms and Related Topics, ed. by S. Ihara, RIMS kokyuroku, 617 (1987), 49-65.

[ 3 ] U. Christian, Untersuchung Selbergscher Zetafunktionen, SFB Heft, 28 (1988).

[ 4 ] — Z Zur Berechnung des Ranges der Schar der Elliptischen Spitzenformen, SFB Heft, 34 (1988).

[5] D. A. Hejhal, The Selberg trace formula for PSL $(2, R)$ Vol. 1, Lecture Notes in Math., Springer, no. 548, (1976).

[ 6 ] — , The Selberg trace formula for PSL $(2, R)$ Vol. 2, Lecture Notes in Math., Springer, no. 1001, (1983).

[ 7 ] T. Hiramatsu, On some dimension formula for automorphic forms of weight one II, Nagoya Math. J., 105 (1987), 169-186.

[ 8 ] T. Hiramatsu and S. Akiyama, On some dimension formula for automorphic forms of weight one III, Nagoya Math. J., 111 (1988), 157-163.

[ 9 ] H. Ishikawa, On the trace formula for Hecke operators, J. Fac. Sci. Univ. Tokyo, Sec. IA, 20, no. 2, (1973), 217-238.

[10] T. Kubota, Elementary theory of Eisenstein series, Kodansha and John Wiley, Tokyo and New York, 1973. 
[11] A. Selberg, Harmonic analysis and discontinuous groups on weakly symmetric Riemannian spaces with applications to Dirichlet series, J. Indian Math. Soc., 20 (1956), 47-87.

[12] Y. Tanigawa and H. Ishikawa, The dimension formula of cusp forms of weight one for $\Gamma_{0}(p)$, Nagoya Math. J., 111 (1988), 115-129.

S. Akiyama

Department of Mathematical Science

Graduate School of Science and Technology

Niigata University

Niigata, 950-21

Japan

Y. Tanigawa

Department of Mathematics

School of Science

Nagoya University

Chikusa-ku, Nagoya 464-01

Japan 